\title{
SCREENINGS AND VERTEX OPERATORS OF QUANTUM SUPERALGEBRA $U_{q}(\widehat{s l}(N \mid 1))$
}

\author{
September 21, 2018 \\ TAKEO KOJIMA \\ Faculty of Engineering, Yamagata University, Jonan 4-3-16, Yonezawa 992-8510, Japan \\ kojima@yz.yamagata-u.ac.jp
}

\begin{abstract}
We construct the screening currents of the quantum superalgebra $U_{q}(\widehat{s l}(N \mid 1))$ for an arbitrary level $k \neq-N+1$. We show that these screening currents commute with the superalgebra modulo total difference. We propose bosonizations of the vertex operators by using the screening currents. We check that these vertex operators are the intertwiners among the Fock-Wakimoto representation and the typical representation for rank $N \leq 4$.
\end{abstract}




\section{Introduction}

Bosonizations are known to be a powerful method to construct correlation functions not only in conformal field theory [1, but also in exactly solvable lattice model [2]. In the previous paper [3] we constructed a bosonization of the quantum affine superalgebra $U_{q}(\widehat{s l}(N \mid 1))$ for an arbitrary level $k \in \mathbf{C}$. Bosonizations for an arbitrary level $k \in \mathbf{C}$ [5, 6, 7, 8, 9, 10, 11, 3, are completely different from those of level $k=1$ 12, 13, 14, 15, 16, 17, 18, 19, 20, 21]. This paper is a continuation of the paper [3. In this paper we focus our attention on the screening currents, that play an important role in level $k$ bosonizations $[3]$. We construct the screening currents that commute with the quantum superalgebra $U_{q}(\widehat{s l}(N \mid 1))$ modulo total difference, for an arbitrary level $k \neq-N+1$. Using the screening currents, we construct the screening operators that commute with the quantum superalgebra. The screening currents are useful to study level $k$ bosonizations, that isn't irreducible representation. For instance, (1) the screening currents balance the "background charge" of the vertex operators [7, 11, 23, 24, 25, and (2) the irreducible representation is constructed from the Felder complex by the screening currents [26, 27, 28, 24, 29. In this paper we focus our attention on the background charge problem. We propose bosonizations of the vertex operators [30] that are the intertwiners among the Fock-Wakimoto module and the typical representation, by using the screening operators. We check the intertwining property of these bosonizations of the vertex operators for rank $N \leq 4$. The screening currents and the vertex operators have been constructed only for $U_{q}(\widehat{s l}(N))$, $U_{q}(\widehat{s l}(2 \mid 1))[7,[8,9,11$ by now. This paper gives a higher-rank generalization of the screenings and the vertex operators in $U_{q}(\widehat{s l}(2 \mid 1))$ paper [1]. The representation theories of the superalgebra are much more complicated than non-superalgebra and have rich structures [31, 32, 33, 36].

This paper is organized as follows. In section 2 we recall the Chevalley realization and the Drinfeld realization of the quantum affine superalgebra $U_{q}(\widehat{s l}(N \mid 1))$. In section 3 we give the bosonization of the quantum affine superalgebra $U_{q}(\widehat{s l}(N \mid 1))$ for an arbitrary level $k$. We propose the Fock-Wakimoto module by the $\xi$ - $\eta$ system. In section 4 we introduce the screening currents that commute with the superalgebra modulo total difference, for an arbitrary level $k \neq-N+1$. In section 5 we propose bosonizations of the vertex operators of $U_{q}(\widehat{s l}(N \mid 1))$. We give the level-zero representation of the Drinfeld generators for $U_{q}(\widehat{s l}(3 \mid 1))$ in this section (resp. $U_{q}(\widehat{s l}(4 \mid 1))$ in appendix B). We check that the vertex operators are the intertwiners among the Fock-Wakimoto realization and the typical representation of the quantum superalgebra $U_{q}(\widehat{s l}(N \mid 1))$ for small rank $N \leq 4$. We show non-vanishing property of the correlation functions. In appendix A we summarize useful formulae of the normal orderings. In appendix B we summarize the level-zero representation of the Drinfeld generators for $U_{q}(\widehat{s l}(4 \mid 1))$.

\section{Quantum affine superalgebra $U_{q}(\widehat{s l}(N \mid 1))$}

In this section we recall the definition of the quantum superalgebra $U_{q}(\widehat{s l}(N \mid 1))$. Throughout this paper we fix a complex number $0<|q|<1$. 


\subsection{Chevalley generator}

We recall the definition of the quantum superalgebra $U_{q}(\widehat{s l}(N \mid 1))(N=2,3, \cdots)$ in terms of the Chevalley generators 34 . The Cartan matrix of the affine superalgebra $\widehat{s l}(N \mid 1)$ is given by

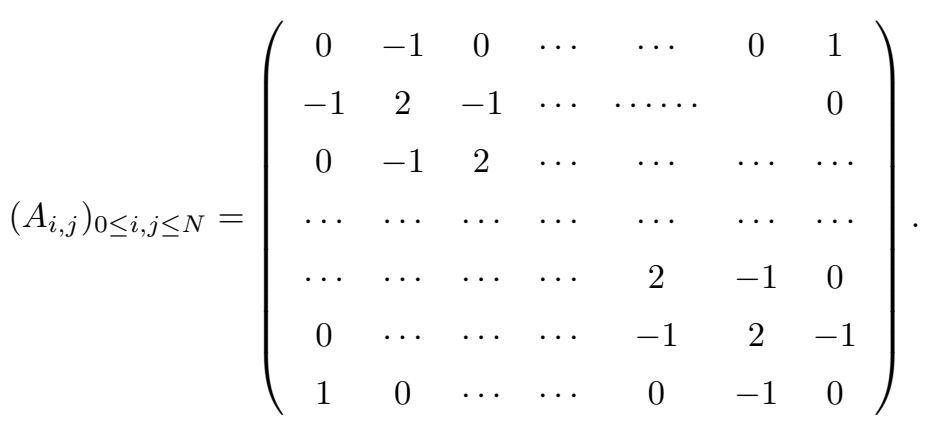

We introduce the orthonormal basis $\left\{\epsilon_{i} \mid i=1,2, \cdots, N+1\right\}$ with the bilinear form, $\left(\epsilon_{i} \mid \epsilon_{j}\right)=\nu_{i} \delta_{i, j}$, where $\nu_{j}=+(j=1,2, \cdots, N)$ and $\nu_{N+1}=-$. Define $\bar{\epsilon}_{i}=\epsilon_{i}-\frac{\nu_{i}}{N-1} \sum_{j=1}^{N+1} \epsilon_{j}$. Note that $\sum_{j=1}^{N} \bar{\epsilon}_{j}=0$. The classical simple roots $\bar{\alpha}_{i}$ and the classical fundamental weights $\bar{\Lambda}_{i}$ are defined by $\bar{\alpha}_{i}=\nu_{i} \epsilon_{i}-\nu_{i+1} \epsilon_{i+1}$, $\bar{\Lambda}_{i}=\sum_{j=1}^{i} \bar{\epsilon}_{j}(1 \leq i \leq N)$. Introduce the affine weight $\Lambda_{0}$ and the null root $\delta$ satisfying $\left(\Lambda_{0} \mid \Lambda_{0}\right)=$ $(\delta \mid \delta)=0,\left(\Lambda_{0} \mid \delta\right)=1,\left(\Lambda_{0} \mid \epsilon_{i}\right)=0,\left(\delta \mid \epsilon_{i}\right)=0,(1 \leq i \leq N)$. The other affine weights and the affine roots are given by $\alpha_{0}=\delta-\sum_{j=1}^{N} \bar{\alpha}_{j}, \alpha_{i}=\bar{\alpha}_{i}, \Lambda_{i}=\bar{\Lambda}_{i}+\Lambda_{0},(1 \leq i \leq N)$. Let $P=\oplus_{j=1}^{N} \mathbf{Z} \Lambda_{j} \oplus \mathbf{Z} \delta$ and $P^{*}=\oplus_{j=1}^{N} \mathbf{Z} h_{j} \oplus \mathbf{Z} d$ the affine $\widehat{s l}(N \mid 1)$ weight lattice and its dual lattice, respectively.

Definition 2.1 34] The quantum affine superalgebra $U_{q}(\widehat{s l}(N \mid 1))$ are generated by the Chevalley generators $h_{i}, e_{i}, f_{i}(1 \leq i \leq N)$. The $\mathbf{Z}_{2}$-grading of the generators are $\left|e_{0}\right|=\left|f_{0}\right|=\left|e_{N}\right|=\left|f_{N}\right|=1$ and zero otherwise. The defining relations are

$$
\left[h_{i}, h_{j}\right]=0, \quad\left[h_{i}, e_{j}\right]=A_{i, j} e_{j}, \quad\left[h_{i}, f_{j}\right]=-A_{i, j} f_{j}, \quad\left[e_{i}, f_{j}\right]=\delta_{i, j} \frac{q^{h_{i}}-q^{-h_{i}}}{q-q^{-1}},
$$

and the Serre relations

$$
\left[e_{j},\left[e_{j}, e_{i}\right]_{q^{-1}}\right]_{q}=0, \quad\left[f_{j},\left[f_{j}, f_{i}\right]_{q^{-1}}\right]_{q}=0 \text { for }\left|A_{i, j}\right|=1, i \neq 0, N
$$

Here and throughout this paper, we use the notations

$$
[X, Y]_{\xi}=X Y-(-1)^{|X||Y|} \xi Y X
$$

We write $[X, Y]_{1}$ as $[X, Y]$ for simplicity. The quantum affine superalgebra $U_{q}(\widehat{s l}(N \mid 1))$ has the $\mathbf{Z}_{2}$-graded Hopf-algebra structure. We take the following coproduct

$$
\Delta\left(e_{i}\right)=e_{i} \otimes 1+q^{h_{i}} \otimes e_{i}, \quad \Delta\left(f_{i}\right)=f_{i} \otimes q^{-h_{i}}+1 \otimes f_{i}, \quad \Delta\left(h_{i}\right)=h_{i} \otimes 1+1 \otimes h_{i},
$$

and the antipode

$$
S\left(e_{i}\right)=-q^{-h_{i}} e_{i}, \quad S\left(f_{i}\right)=-f_{i} q^{h_{i}}, \quad S\left(h_{i}\right)=-h_{i} .
$$


The coproduct $\Delta$ satisfies an algebra automorphism $\Delta(X Y)=\Delta(X) \Delta(Y)$ and the antipode $S$ satisfies a $\mathbf{Z}_{2}$-graded algebra anti-automorphism $S(X Y)=(-1)^{|X||Y|} S(Y) S(X)$. The multiplication rule for the tensor product is $\mathbf{Z}_{2}$-graded and is defined for homogeneous elements $X, Y, X^{\prime}, Y^{\prime} \in U_{q}(\widehat{s l}(N \mid 1))$ and $v \in V, w \in W$ by $X \otimes Y \cdot X^{\prime} \otimes Y^{\prime}=(-1)^{|Y|\left|X^{\prime}\right|} X X^{\prime} \otimes Y Y^{\prime}$ and $X \otimes Y \cdot v \otimes w=(-1)^{|Y||v|} X v \otimes Y w$, which extends to inhomogeneous elements through linearity.

We sometimes use the anti-commutator $\{X, Y\}=X Y+Y X=[X, Y]_{1}$ for $|X|=|Y|=1$.

\subsection{Drinfeld realization}

We recall the Drinfeld's second realization of the quantum affine superalgebra $U_{q}(\widehat{s l}(N \mid 1))$ [34, [35]. The Drinfeld realization is convenient for constructions of bosonizations. We use the standard symbol of $q$-integer

$$
[a]=\frac{q^{a}-q^{-a}}{q-q^{-1}} .
$$

Definition 2.2 34] The Drinfeld generators of the quantum affine superalgebra $U_{q}(\widehat{s l}(N \mid 1))$ are $X_{i, m}^{ \pm}, h_{i, m}, c(1 \leq i \leq N, m \in \mathbf{Z})$. The $\mathbf{Z}_{2}$-grading of the Drinfeld generators are $:\left|X_{N, m}^{ \pm}\right|=1$ $(m \in \mathbf{Z})$ and zero otherwise. Defining relations are

$$
\begin{aligned}
& c: \text { central, }\left[h_{i}, h_{j, m}\right]=0, \\
& {\left[h_{i, m}, h_{j, n}\right]=\frac{\left[A_{i, j} m\right][\mathrm{cm}]}{m} \delta_{m+n, 0} \quad(m, n \neq 0),} \\
& {\left[h_{i}, X_{j}^{ \pm}(z)\right]= \pm A_{i, j} X_{j}^{ \pm}(z),} \\
& {\left[h_{i, m}, X_{j}^{+}(z)\right]=\frac{\left[A_{i, j} m\right]}{m} q^{-\frac{c}{2}|m|} z^{m} X_{j}^{+}(z) \quad(m \neq 0),} \\
& {\left[h_{i, m}, X_{j}^{-}(z)\right]=-\frac{\left[A_{i, j} m\right]}{m} q^{\frac{c}{2}|m|} z^{m} X_{j}^{-}(z) \quad(m \neq 0),} \\
& \left(z_{1}-q^{ \pm A_{i, j}} z_{2}\right) X_{i}^{ \pm}\left(z_{1}\right) X_{j}^{ \pm}\left(z_{2}\right)=\left(q^{ \pm A_{j, i}} z_{1}-z_{2}\right) X_{j}^{ \pm}\left(z_{2}\right) X_{i}^{ \pm}\left(z_{1}\right) \quad \text { for }\left|A_{i, j}\right| \neq 0 \\
& {\left[X_{i}^{ \pm}\left(z_{1}\right), X_{j}^{ \pm}\left(z_{2}\right)\right]=0 \quad \text { for }\left|A_{i, j}\right|=0} \\
& {\left[X_{i}^{+}\left(z_{1}\right), X_{j}^{-}\left(z_{2}\right)\right]=\frac{\delta_{i, j}}{\left(q-q^{-1}\right) z_{1} z_{2}}\left(\delta\left(q^{-c} z_{1} / z_{2}\right) \Psi_{i}^{+}\left(q^{\frac{c}{2}} z_{2}\right)-\delta\left(q^{c} z_{1} / z_{2}\right) \Psi_{i}^{-}\left(q^{-\frac{c}{2}} z_{2}\right)\right),} \\
& {\left[X_{i}^{ \pm}\left(z_{1}\right),\left[X_{i}^{ \pm}\left(z_{2}\right), X_{j}^{ \pm}(z)\right]_{q^{-1}}\right]_{q}+\left(z_{1} \leftrightarrow z_{2}\right)=0 \quad \text { for }\left|A_{i, j}\right|=1, i \neq N .}
\end{aligned}
$$

where we have used $\delta(z)=\sum_{m \in \mathbf{Z}} z^{m}$. Here we have used the abbreviation $h_{i}=h_{i, 0}$. We have used the generating function

$$
\begin{aligned}
& X_{j}^{ \pm}(z)=\sum_{m \in \mathbf{Z}} X_{j, m}^{ \pm} z^{-m-1}, \\
& \Psi_{i}^{+}(z)=q^{h_{i}} \exp \left(\left(q-q^{-1}\right) \sum_{m>0} h_{i, m} z^{-m}\right) \\
& \Psi_{i}^{-}(z)=q^{-h_{i}} \exp \left(-\left(q-q^{-1}\right) \sum_{m>0} h_{i,-m} z^{m}\right) .
\end{aligned}
$$


The relations between the Chevalley generators and the Drinfeld realization are given by

$$
\begin{aligned}
h_{i} & =h_{i, 0}, \quad e_{i}=X_{i, 0}^{+}, \quad f_{i}=X_{i, 0}^{-} \quad \text { for } 1 \leq i \leq N, \\
h_{0} & =c-\left(h_{1,0}+\cdots+h_{N, 0}\right) \\
e_{0} & =(-1)\left[X_{N, 0}^{-} \cdots,\left[X_{3,0}^{-},\left[X_{2,0}^{-}, X_{1,1}^{-}\right]_{q^{-1}}\right]_{q^{-1}} \cdots\right]_{q^{-1}} q^{-h_{1,0}-h_{2,0}-\cdots-h_{N, 0}}, \\
f_{0} & =q^{h_{1,0}+h_{2,0}+\cdots+h_{N, 0}}\left[\cdots\left[\left[X_{1,-1}^{+}, X_{2,0}^{+}\right]_{q}, X_{3,0}^{+}\right]_{q}, \cdots X_{N, 0}^{+}\right]_{q} .
\end{aligned}
$$

\section{Bosonization of $U_{q}(\widehat{s l}(N \mid 1))$}

In this section we recall the bosonization of $U_{q}(\widehat{s l}(N \mid 1))$ for an arbitrary level $k \in \mathbf{C}[3]$.

\subsection{Boson}

We introduce the bosons and the zero-mode operators $a_{m}^{j}, Q_{a}^{j}(m \in \mathbf{Z}, 1 \leq j \leq N), b_{m}^{i, j}, Q_{b}^{i, j}, c_{m}^{i, j}, Q_{c}^{i, j}$ $(m \in \mathbf{Z}, 1 \leq i<j \leq N+1)$. The bosons $a_{m}^{i}, b_{m}^{i, j}, c_{m}^{i, j},\left(m \in \mathbf{Z}_{\neq 0}\right)$ and the zero-mode operators $a_{0}^{i}, Q_{a}^{i}$, $b_{0}^{i, j}, Q_{b}^{i, j}, c_{0}^{i, j}, Q_{c}^{i, j}$ that satisfy

$$
\begin{aligned}
& {\left[a_{m}^{i}, a_{n}^{j}\right]=\frac{[(k+N-1) m]\left[A_{i, j} m\right]}{m} \delta_{m+n, 0}, \quad\left[a_{0}^{i}, Q_{a}^{j}\right]=(k+N-1) A_{i, j},} \\
& {\left[b_{m}^{i, j}, b_{n}^{i^{\prime}, j^{\prime}}\right]=-\nu_{i} \nu_{j} \frac{[m]^{2}}{m} \delta_{i, i^{\prime}} \delta_{j, j^{\prime}} \delta_{m+n, 0}, \quad\left[b_{0}^{i, j}, Q_{b}^{i^{\prime}, j^{\prime}}\right]=-\nu_{i} \nu_{j} \delta_{i, i^{\prime}} \delta_{j, j^{\prime}},} \\
& {\left[c_{m}^{i, j}, c_{n}^{i^{\prime}, j^{\prime}}\right]=\nu_{i} \nu_{j} \frac{[m]^{2}}{m} \delta_{i, i^{\prime}} \delta_{j, j^{\prime}} \delta_{m+n, 0}, \quad\left[c_{0}^{i, j}, Q_{c}^{i^{\prime}, j^{\prime}}\right]=\nu_{i} \nu_{j} \delta_{i, i^{\prime}} \delta_{j, j^{\prime}},}
\end{aligned}
$$

and other commutators vanish. We impose the cocycle condition on the zero-mode operator $Q_{b}^{i, j}(1 \leq$ $i<j \leq N+1)$ by

$$
\left[Q_{b}^{i, j}, Q_{b}^{i^{\prime}, j^{\prime}}\right]=\delta_{j, N+1} \delta_{j^{\prime}, N+1} \pi \sqrt{-1} \quad \text { for }(i, j) \neq\left(i^{\prime}, j^{\prime}\right)
$$

We have the following (anti)commutation relations

$$
\begin{aligned}
& {\left[\exp \left(Q_{b}^{i, j}\right), \exp \left(Q_{b}^{i^{\prime}, j^{\prime}}\right)\right]=0 \quad\left(1 \leq i<j \leq N, 1 \leq i^{\prime}<j^{\prime} \leq N\right),} \\
& \left\{\exp \left(Q_{b}^{i, N+1}\right), \exp \left(Q_{b}^{j, N+1}\right)\right\}=0 \quad(1 \leq i \neq j \leq N) .
\end{aligned}
$$

In what follows we use the standard normal ordering symbol ::. We set $b^{i, j}(z), c^{i, j}(z), b_{ \pm}^{i, j}(z), a_{ \pm}^{j}(z)$ and $\left(\frac{\gamma_{1}}{\beta_{1}} \frac{\gamma_{2}}{\beta_{2}} \cdots \frac{\gamma_{r}}{\beta_{r}} a^{i}\right)(z \mid \alpha)$ by

$$
\begin{aligned}
& a_{ \pm}^{j}(z)= \pm\left(q-q^{-1}\right) \sum_{ \pm m>0} a_{m}^{j} z^{-m} \pm a_{0}^{j} \log q \\
& b^{i, j}(z)=-\sum_{m \neq 0} \frac{b_{m}^{i, j}}{[m]} z^{-m}+Q_{b}^{i, j}+b_{0}^{i, j} \log z \\
& b_{ \pm}^{i, j}(z)= \pm\left(q-q^{-1}\right) \sum_{ \pm m>0} b_{m}^{i, j} z^{-m} \pm b_{0}^{i, j} \log q \\
& c^{i, j}(z)=-\sum_{m \neq 0} \frac{c_{m}^{i, j}}{[m]} z^{-m}+Q_{c}^{i, j}+c_{0}^{i, j} \log z
\end{aligned}
$$




$$
\left(\frac{\gamma_{1}}{\beta_{1}} \frac{\gamma_{2}}{\beta_{2}} \cdots \frac{\gamma_{r}}{\beta_{r}} a^{i}\right)(z \mid \alpha)=-\sum_{m \neq 0} \frac{\left[\gamma_{1} m\right] \cdots\left[\gamma_{r} m\right]}{\left[\beta_{1} m\right] \cdots\left[\beta_{r} m\right]} \frac{a_{m}^{i}}{[m]} q^{-\alpha|m|} z^{-m}+\frac{\gamma_{1} \cdots \gamma_{r}}{\beta_{1} \cdots \beta_{r}}\left(Q_{a}^{i}+a_{0}^{i} \log z\right)
$$

\section{$3.2 \quad$ Bosonization of $U_{q}(\widehat{s l}(N \mid 1))$}

We recall the bosonizations of the quantum superalgebra $U_{q}(\widehat{s l}(N \mid 1))$.

Theorem 3.1 [3] A bosonization of the quantum affine superalgebra $U_{q}(\widehat{s l}(N \mid 1))$ for an arbitrary level $k \in \mathbf{C}$ is given as follows. For $1 \leq i \leq N-1$ we set

$$
\begin{aligned}
X_{i}^{+}(z) & =\frac{1}{\left(q-q^{-1}\right) z} \sum_{j=1}^{i}\left(X_{i}^{+(j, 1)}(z)-X_{i}^{+(j, 2)}(z)\right) \\
X_{N}^{+}(z) & =q^{N-2} \sum_{j=1}^{N} X_{N}^{+(j, 0)}(z) \\
X_{i}^{-}(z)= & \frac{1}{\left(q-q^{-1}\right) z}\left(\sum_{j=1}^{i-1}\left(X_{i}^{-(j, 1)}(z)-X_{i}^{-(j, 2)}(z)\right)+\left(X_{i}^{-(i, 1)}(z)-X_{i}^{-(i, 2)}(z)\right)\right. \\
X_{N}^{-}(z)= & \left.-\sum_{j=i+1}^{N-1}\left(X_{i}^{-(j, 1)}(z)-X_{i}^{-(j, 2)}(z)\right)\right)+q^{k+N-1} X_{i}^{-(N, 0)}(z) \\
\Psi_{i}^{ \pm}\left(q^{ \pm \frac{k}{2}} z\right)= & \exp \left(a_{ \pm}^{i}\left(q^{ \pm \frac{k+N-1}{2}} z\right)+\sum_{j=1}^{N} q^{-N+j+1}\left(-X_{N}^{l, i+1}\left(q^{ \pm(l, 1)}(z)+X_{N}^{-(j, 2)}(z)\right)\right.\right. \\
& \left.+\sum_{l=i+1}^{N}\left(b_{ \pm}^{i, l}\left(q^{ \pm(k+l)} z\right)-b_{ \pm}^{i-1, l}\left(q^{ \pm(k+l-1)} z\right)\right)+b_{ \pm}^{i, N+1}\left(q^{ \pm(k+N)} z\right)-b_{ \pm}^{i+1, N+1}\left(q^{ \pm(k+N-1)} z\right)\right) \\
\Psi_{N}^{ \pm}\left(q^{ \pm \frac{k}{2}} z\right)= & \exp \left(a_{ \pm}^{N}\left(q^{ \pm \frac{k+N-1}{2}} z\right)-\sum_{l=1}^{N-1}\left(b_{ \pm}^{l, N}\left(q^{ \pm(k+l)} z\right)+b_{ \pm}^{l, N+1}\left(q^{ \pm(k+l)} z\right)\right)\right)
\end{aligned}
$$

Here we have used the auxiliary bosonic operators $X_{i}^{ \pm(j, s)}(z)$ as follows.

For $1 \leq i \leq N-1$ and $1 \leq j \leq i$ we set

$$
\begin{aligned}
X_{i}^{+(j, 1)}(z)= & : \exp \left((b+c)^{j, i}\left(q^{j-1} z\right)+b_{+}^{j, i+1}\left(q^{j-1} z\right)-(b+c)^{j, i+1}\left(q^{j} z\right)\right. \\
& \left.+\sum_{l=1}^{j-1}\left(b_{+}^{l, i+1}\left(q^{l-1} z\right)-b_{+}^{l, i}\left(q^{l} z\right)\right)\right): \\
X_{i}^{+(j, 2)}(z)= & : \exp \left((b+c)^{j, i}\left(q^{j-1} z\right)+b_{-}^{j, i+1}\left(q^{j-1} z\right)-(b+c)^{j, i+1}\left(q^{j-2} z\right)\right. \\
& \left.+\sum_{l=1}^{j-1}\left(b_{+}^{l, i+1}\left(q^{l-1} z\right)-b_{+}^{l, i}\left(q^{l} z\right)\right)\right):
\end{aligned}
$$

For $1 \leq j \leq N$ we set

$$
X_{N}^{+(j, 0)}(z)=: \exp \left((b+c)^{j, N}\left(q^{j-1} z\right)+b^{j, N+1}\left(q^{j-1} z\right)-\sum_{l=1}^{j-1}\left(b_{+}^{l, N+1}\left(q^{l} z\right)+b_{+}^{l, N}\left(q^{l} z\right)\right)\right): .
$$


For $1 \leq i \leq N-1$ and $1 \leq j \leq i-1$ we set

$$
\begin{aligned}
X_{i}^{-(j, 1)}(z)= & : \exp \left(a_{-}^{i}\left(q^{-\frac{k+N-1}{2}} z\right)+(b+c)^{j, i+1}\left(q^{-k-j} z\right)-b_{-}^{j, i}\left(q^{-k-j} z\right)-(b+c)^{j, i}\left(q^{-k-j+1} z\right)\right. \\
& +\sum_{l=j+1}^{i}\left(b_{-}^{l, i+1}\left(q^{-k-l+1} z\right)-b_{-}^{l, i}\left(q^{-k-l} z\right)\right)+\sum_{l=i+1}^{N}\left(b_{-}^{i, l}\left(q^{-k-l} z\right)-b_{-}^{i+1, l}\left(q^{-k-l+1} z\right)\right) \\
& \left.+b_{-}^{i, N+1}\left(q^{-k-N} z\right)-b_{-}^{i+1, N+1}\left(q^{-k-N+1} z\right)\right): \\
X_{i}^{-(j, 2)}(z)= & : \exp \left(a_{-}^{i}\left(q^{-\frac{k+N-1}{2}} z\right)+(b+c)^{j, i+1}\left(q^{-k-j} z\right)-b_{+}^{j, i}\left(q^{-k-j} z\right)-(b+c)^{j, i}\left(q^{-k-j-1} z\right)\right. \\
& +\sum_{l=j+1}^{i}\left(b_{-}^{l, i+1}\left(q^{-k-l+1} z\right)-b_{-}^{l, i}\left(q^{-k-l} z\right)\right)+\sum_{l=i+1}^{N}\left(b_{-}^{i, l}\left(q^{-k-l} z\right)-b_{-}^{i+1, l}\left(q^{-k-l+1} z\right)\right) \\
& \left.+b_{-}^{i, N+1}\left(q^{-k-N} z\right)-b_{-}^{i+1, N+1}\left(q^{-k-N+1} z\right)\right):
\end{aligned}
$$

For $1 \leq i \leq N-1$ we set

$$
\begin{aligned}
X_{i}^{-(i, 1)}(z)= & : \exp \left(a_{-}^{i}\left(q^{-\frac{k+N-1}{2}} z\right)+(b+c)^{i, i+1}\left(q^{-k-i} z\right)+\sum_{l=i+1}^{N}\left(b_{-}^{i, l}\left(q^{-k-l} z\right)-b_{-}^{i+1, l}\left(q^{-k-l+1} z\right)\right)\right. \\
& \left.+b_{-}^{i, N+1}\left(q^{-k-N} z\right)-b_{-}^{i+1, N+1}\left(q^{-k-N+1} z\right)\right): \\
X_{i}^{-(i, 2)}(z)= & : \exp \left(a_{+}^{i}\left(q^{\frac{k+N-1}{2}} z\right)+(b+c)^{i, i+1}\left(q^{k+i} z\right)+\sum_{l=i+1}^{N}\left(b_{+}^{i, l}\left(q^{k+l} z\right)-b_{+}^{i+1, l}\left(q^{k+l-1} z\right)\right)\right. \\
& \left.+b_{+}^{i, N+1}\left(q^{k+N} z\right)-b_{+}^{i+1, N+1}\left(q^{k+N-1} z\right)\right):
\end{aligned}
$$

For $1 \leq i \leq N-1$ and $i+1 \leq j \leq N-1$ we set

$$
\begin{aligned}
X_{i}^{-(j, 1)}(z)= & : \exp \left(a_{+}^{i}\left(q^{\frac{k+N-1}{2}} z\right)+(b+c)^{i, j+1}\left(q^{k+j} z\right)+b_{+}^{i+1, j+1}\left(q^{k+j} z\right)-(b+c)^{i+1, j+1}\left(q^{k+j+1} z\right)\right. \\
& \left.+\sum_{l=j+1}^{N}\left(b_{+}^{i, l}\left(q^{k+l} z\right)-b_{+}^{i+1, l}\left(q^{k+l-1} z\right)\right)+b_{+}^{i, N+1}\left(q^{k+N} z\right)-b_{+}^{i+1, N+1}\left(q^{k+N-1} z\right)\right):,(3.25) \\
X_{i}^{-(j, 2)}(z)= & : \exp \left(a_{+}^{i}\left(q^{\frac{k+N-1}{2}} z\right)+(b+c)^{i, j+1}\left(q^{k+j} z\right)+b_{-}^{i+1, j+1}\left(q^{k+j} z\right)-(b+c)^{i+1, j+1}\left(q^{k+j-1} z\right)\right. \\
& \left.+\sum_{l=j+1}^{N}\left(b_{+}^{i, l}\left(q^{k+l} z\right)-b_{+}^{i+1, l}\left(q^{k+l-1} z\right)\right)+b_{+}^{i, N+1}\left(q^{k+N} z\right)-b_{+}^{i+1, N+1}\left(q^{k+N-1} z\right)\right): .(3.26)
\end{aligned}
$$

For $1 \leq i \leq N-1$ we set

$$
X_{i}^{-(N, 0)}(z)=: \exp \left(a_{+}^{i}\left(q^{\frac{k+N-1}{2}} z\right)-b^{i, N+1}\left(q^{k+N-1} z\right)-b_{+}^{i+1, N+1}\left(q^{k+N-1} z\right)+b^{i+1, N+1}\left(q^{k+N} z\right)\right): .
$$

For $1 \leq j \leq N-1$ we set

$$
\begin{aligned}
X_{N}^{-(j, 1)}(z)= & : \exp \left(a_{-}^{N}\left(q^{-\frac{k+N-1}{2}} z\right)-b_{-}^{j, N}\left(q^{-k-j} z\right)-(b+c)^{j, N}\left(q^{-k-j+1} z\right)\right. \\
& \left.-b_{-}^{j, N+1}\left(q^{-k-j} z\right)-b^{j, N+1}\left(q^{-k-j+1} z\right)-\sum_{l=j+1}^{N-1}\left(b_{-}^{l, N}\left(q^{-k-l} z\right)+b_{-}^{l, N+1}\left(q^{-k-l} z\right)\right)\right): \\
X_{N}^{-(j, 2)}(z)= & : \exp \left(a_{-}^{N}\left(q^{-\frac{k+N-1}{2}} z\right)-b_{+}^{j, N}\left(q^{-k-j} z\right)-(b+c)^{j, N}\left(q^{-k-j-1} z\right)\right.
\end{aligned}
$$




$$
\begin{aligned}
& \left.-b_{+}^{j, N+1}\left(q^{-k-j} z\right)-b^{j, N+1}\left(q^{-k-j-1} z\right)-\sum_{l=j+1}^{N-1}\left(b_{-}^{l, N}\left(q^{-k-l} z\right)+b_{-}^{l, N+1}\left(q^{-k-l} z\right)\right)\right):, \\
X_{N}^{-(N, 1)}(z)= & : \exp \left(a_{-}^{N}\left(q^{-\frac{k+N-1}{2}} z\right)-b^{N, N+1}\left(q^{-k-N+1} z\right)\right): \\
X_{N}^{-(N, 2)}(z)= & : \exp \left(a_{+}^{N}\left(q^{\frac{k+N-1}{2}} z\right)-b^{N, N+1}\left(q^{k+N-1} z\right)\right): .
\end{aligned}
$$

The $\mathbf{Z}_{2}$-grading is : $\left|X_{N}^{ \pm(j, s)}(z)\right|=1$ and zero otherwise.

Very explicitly, we have

$$
\begin{aligned}
h_{i, m}= & q^{-\frac{N-1}{2}|m|} a_{m}^{i}+\sum_{l=1}^{i}\left(q^{-\left(\frac{k}{2}+l-1\right)|m|} b_{m}^{l, i+1}-q^{-\left(\frac{k}{2}+l\right)|m|} b_{m}^{l, i}\right) \\
& +\sum_{l=i+1}^{N}\left(q^{-\left(\frac{k}{2}+l\right)|m|} b_{m}^{i, l}-q^{-\left(\frac{k}{2}+l-1\right)|m|} b_{m}^{i+1, l}\right) \\
& +q^{-\left(\frac{k}{2}+N\right)|m|} b_{m}^{i, N+1}-q^{-\left(\frac{k}{2}+N-1\right)|m|} b_{m}^{i+1, N+1} \quad(1 \leq i \leq N-1), \\
h_{N, m}= & q^{-\frac{N-1}{2}|m|} a_{m}^{N}-\sum_{l=1}^{N-1}\left(q^{-\left(\frac{k}{2}+l\right)|m|} b_{m}^{l, N}+q^{-\left(\frac{k}{2}+l\right)|m|} b_{m}^{l, N+1}\right) .
\end{aligned}
$$

\subsection{Fock-Wakimoto module}

We introduce the vacuum state $|0\rangle$ of the boson Fock space by

$$
a_{m}^{i}|0\rangle=b_{m}^{i, j}|0\rangle=c_{m}^{i, j}|0\rangle=0 \quad(m \geq 0) .
$$

For $p_{a}^{i} \in \mathbf{C}(1 \leq i \leq N), p_{b}^{i, j} \in \mathbf{C}(1 \leq i<j \leq N+1), p_{c}^{i, j} \in \mathbf{C}(1 \leq i<j \leq N)$, we set

$$
\begin{aligned}
& \left|p_{a}, p_{b}, p_{c}\right\rangle \\
= & \exp \left(\sum_{i, j=1}^{N} \frac{\operatorname{Min}(i, j)(N-1-\operatorname{Max}(i, j))}{(N-1)(k+N-1)} p_{a}^{i} Q_{a}^{j}-\sum_{1 \leq i<j \leq N+1} p_{b}^{i, j} Q_{b}^{i, j}+\sum_{1 \leq i<j \leq N} p_{c}^{i, j} Q_{c}^{i, j}\right)|0\rangle .
\end{aligned}
$$

It satisfies

$$
a_{0}^{i}\left|p_{a}, p_{b}, p_{c}\right\rangle=p_{a}^{i}\left|p_{a}, p_{b}, p_{c}\right\rangle, b_{0}^{i, j}\left|p_{a}, p_{b}, p_{c}\right\rangle=p_{b}^{i, j}\left|p_{a}, p_{b}, p_{c}\right\rangle, c_{0}^{i, j}\left|p_{a}, p_{b}, p_{c}\right\rangle=p_{c}^{i, j}\left|p_{a}, p_{b}, p_{c}\right\rangle
$$

The boson Fock space $F\left(p_{a}, p_{b}, p_{c}\right)$ is generated by the bosons $a_{m}^{i}, b_{m}^{i, j}, c_{m}^{i, j}$ on the vector $\left|p_{a}, p_{b}, p_{c}\right\rangle$. We set the space $F\left(p_{a}\right)$ by

$$
F\left(p_{a}\right)=\bigoplus_{\substack{i, j \\ p_{b}^{i, j}=-p_{c}^{i, j} \in \mathbf{Z} \\ p_{b}^{i, N+1} \in \mathbf{Z} \\(1 \leq i \leq i \leq N)}} F\left(p_{a}, p_{b}, p_{c}\right) .
$$

We impose the restriction $p_{b}^{i, j}=-p_{c}^{i, j} \in \mathbf{Z}(1 \leq i<j \leq N)$, because the $X_{i, m}^{ \pm}$change $Q_{b}^{i, j}+Q_{c}^{i, j}$. The $F\left(p_{a}\right)$ is $U_{q}(\widehat{s l}(N \mid 1))$-module. We set the vector $|\lambda\rangle=\left|p_{a}, 0,0\right\rangle$ upon the specialization $p_{b}^{i, j}=0(1 \leq i<$ $j \leq N+1)$ and $p_{c}^{i, j}=0(1 \leq i<j \leq N)$. 
Proposition 3.2 The $|\lambda\rangle=\left|p_{a}, 0,0\right\rangle$ is the highest weight vector of the highest weight whose classical part is $\bar{\lambda}=\sum_{j=1}^{N} p_{a}^{j} \bar{\Lambda}_{j}$.

$$
\begin{array}{ll}
h_{i, m}|\lambda\rangle=0, & X_{i, m}^{ \pm}|\lambda\rangle=0, \quad(m>0), \\
X_{i, 0}^{+}|\lambda\rangle=0, & h_{i, 0}|\lambda\rangle=p_{a}^{i}|\lambda\rangle .
\end{array}
$$

Using the highest weight vector $|\lambda\rangle$, we have the highest weight module $V(\lambda)$ of $U_{q}(\widehat{s l}(N \mid 1))$.

$$
V(\lambda) \subset F\left(p_{a}\right) .
$$

The module $F\left(p_{a}\right)$ is not irreducible. We recall the non-quantum algebra $\widehat{s l}(2)$ case [27]. The irreducible highest weight module $L(\lambda)$ for the affine algebra $\widehat{s l}(2)$ was constructed from the Fock-Wakimoto module on the boson Fock space [5] by the Felder complex. We recall the quantum algebra $U_{q}(\widehat{s l}(2))$ case $[7,8,24$. The irreducible highest weight module $L(\lambda)$ for $U_{q}(\widehat{s l}(2))$ was constructed from the similar space as $F\left(p_{a}\right)$ by two steps; the first step is the construction of the Fock-Wakimoto module by the $\xi-\eta$ system, and the second step is the resolution by the Felder complex [24]. The submodule of the quantum algebra $U_{q}(\widehat{s l}(2))$, induced by the $\xi-\eta$ system, plays the same role as the Fock-Wakimoto module of the non-quantum algebra $\widehat{s l}(2)$. We call this submodule induced by the $\xi-\eta$ system the "Fock-Wakimoto module". In this paper we study the $\xi-\eta$ system and propose the Fock-Wakimoto module for $U_{q}(\widehat{s l}(N \mid 1))$.

Definition 3.3 We introduce the operators $\xi_{m}^{i, j}$ and $\eta_{m}^{i, j}(1 \leq i<j \leq N, m \in \mathbf{Z})$ by

$$
\eta^{i, j}(z)=\sum_{m \in \mathbf{Z}} \eta_{m}^{i, j} z^{-m-1}=: e^{c^{i, j}(z)}:, \quad \xi^{i, j}(z)=\sum_{m \in \mathbf{Z}} \xi_{m}^{i, j} z^{-m}=: e^{-c^{i, j}(z)}: .
$$

The Fourier components $\eta_{m}^{i, j}=\oint \frac{d z}{2 \pi \sqrt{-1}} z^{m} \eta^{i, j}(z), \xi_{m}^{i, j}=\oint \frac{d z}{2 \pi \sqrt{-1}} z^{m-1} \xi^{i, j}(z)(m \in \mathbf{Z})$ are well defined on the space $F\left(p_{a}\right)$. They satisfy the anti-commutation relations.

$$
\left\{\eta_{m}^{i, j}, \xi_{n}^{i, j}\right\}=\delta_{m+n, 0},\left\{\eta_{m}^{i, j}, \eta_{n}^{i, j}\right\}=\left\{\xi_{m}^{i, j}, \xi_{n}^{i, j}\right\}=0 \quad(1 \leq i<j \leq N) .
$$

They commute with each other

$$
\left[\eta_{m}^{i, j}, \xi_{n}^{i^{\prime}, j^{\prime}}\right]=\left[\eta_{m}^{i, j}, \eta_{n}^{i^{\prime}, j^{\prime}}\right]=\left[\xi_{m}^{i, j}, \xi_{n}^{i^{\prime}, j^{\prime}}\right]=0 \quad(i, j) \neq\left(i^{\prime}, j^{\prime}\right)
$$

We focus our attention on the operators $\eta_{0}^{i, j}, \xi_{0}^{i, j}$ satisfying $\left(\eta_{0}^{i, j}\right)^{2}=0,\left(\xi_{0}^{i, j}\right)^{2}=0$. They satisfy

$$
\operatorname{Im}\left(\eta_{0}^{i, j}\right)=\operatorname{Ker}\left(\eta_{0}^{i, j}\right), \quad \operatorname{Im}\left(\xi_{0}^{i, j}\right)=\operatorname{Ker}\left(\xi_{0}^{i, j}\right) .
$$

The products $\eta_{0}^{i, j} \xi_{0}^{i, j}$ and $\xi_{0}^{i, j} \eta_{0}^{i, j}$ are the projection operators, which satisfy

$$
\eta_{0}^{i, j} \xi_{0}^{i, j}+\xi_{0}^{i, j} \eta_{0}^{i, j}=1
$$

and

$$
\left(\eta_{0}^{i, j} \xi_{0}^{i, j}\right)^{2}=\eta_{0}^{i, j} \xi_{0}^{i, j},\left(\xi_{0}^{i, j} \eta_{0}^{i, j}\right)^{2}=\xi_{0}^{i, j} \eta_{0}^{i, j},\left(\xi_{0}^{i, j} \eta_{0}^{i, j}\right)\left(\eta_{0}^{i, j} \xi_{0}^{i, j}\right)=0,\left(\eta_{0}^{i, j} \xi_{0}^{i, j}\right)\left(\xi_{0}^{i, j} \eta_{0}^{i, j}\right)=0 .
$$

Hence we have a direct sum decomposition.

$$
F\left(p_{a}\right)=\eta_{0}^{i, j} \xi_{0}^{i, j} F\left(p_{a}\right) \oplus \xi_{0}^{i, j} \eta_{0}^{i, j} F\left(p_{a}\right),
$$


and

$$
\operatorname{Ker}\left(\eta_{0}^{i, j}\right)=\eta_{0}^{i, j} \xi_{0}^{i, j} F\left(p_{a}\right), \quad \operatorname{Coker}\left(\eta_{0}^{i, j}\right)=\xi_{0}^{i, j} \eta_{0}^{i, j} F\left(p_{a}\right)=F_{\Lambda} /\left(\eta_{0}^{i, j} \xi_{0}^{i, j}\right) F_{\Lambda}
$$

We set

$$
\eta_{0}=\prod_{1 \leq i<j \leq N} \eta_{0}^{i, j}, \quad \xi_{0}=\prod_{1 \leq i<j \leq N} \xi_{0}^{i, j} .
$$

Definition 3.4 We introduce the subspace $\mathcal{F}\left(p_{a}\right)$ by

$$
\mathcal{F}\left(p_{a}\right)=\eta_{0} \xi_{0} F\left(p_{a}\right)
$$

The operators $\eta_{0}^{i, j}, \xi_{0}^{i, j}$ commute with the operators $X_{i^{\prime}, j^{\prime}}^{ \pm}(z), \Psi_{i^{\prime}}^{ \pm}(z)$ up to sign \pm . When we set the operators $\widetilde{X}_{i}^{ \pm}(z), \widetilde{\Psi}_{i}^{ \pm}(z)$ by the conditions $\widetilde{X}_{i}^{ \pm}(z) \eta_{0}^{i^{\prime}, j^{\prime}}=\eta_{0}^{i^{\prime}, j^{\prime}} X_{i}^{ \pm}(z), \widetilde{\Psi}_{i}^{ \pm}(z) \eta_{0}^{i^{\prime}, j^{\prime}}=\eta_{0}^{i^{\prime}, j^{\prime}} \Psi_{i}^{ \pm}(z)$, the bosonic operators $\widetilde{X}_{i}^{ \pm}(z), \widetilde{\Psi}_{i}^{ \pm}(z)$ give a bosonization of $U_{q}(\widehat{s l}(N \mid 1))$ again.

Proposition 3.5 [4] The subspace $\mathcal{F}\left(p_{a}\right)$ is the $U_{q}(\widehat{s l}(N \mid 1))$ module.

We call the submodule $\mathcal{F}\left(p_{a}\right)$ the Fock-Wakimoto module. It is expected that we have the irreducible highest weight module $L(\lambda)$ with the highest weight $\lambda$, whose classical part $\bar{\lambda}=\sum_{j=1}^{N} p_{a}^{i} \bar{\Lambda}_{i}$, by the Felder complex. The construction of the Felder complex is open problem even for non-superalgebra $U_{q}(\widehat{s l}(3))$. We would like to report the Felder complex of $U_{q}(\widehat{s l}(N))$ and $U_{q}(\widehat{s l}(N \mid 1))$ in the future publications.

\section{Screening current}

In this section we introduce the screening operators $S_{i}(i=1,2, \cdots, N)$, which commute with $U_{q}(\widehat{s l}(N \mid 1))$ for an arbitrary level $k \neq-N+1$. We need the screening operators to construct the vertex operators.

\subsection{Screening current}

We set the $q$-difference operators with a parameter $\alpha$ by

$$
\left({ }_{\alpha} \partial_{z} f\right)(z)=\frac{f\left(q^{\alpha} z\right)-f\left(q^{-\alpha} z\right)}{\left(q-q^{-1}\right) z} .
$$

The Jackson integral with parameter $p \in \mathbf{C}(|p|<1)$ and $s \in \mathbf{C}^{*}$ is defined by

$$
\int_{0}^{s \infty} f(z) d_{p} z=s(1-p) \sum_{m \in \mathbf{Z}} f\left(s p^{m}\right) p^{m} .
$$

The Jackson integral satisfies

$$
\int_{0}^{s \infty}\left({ }_{\alpha} \partial_{z} f\right)(z) d_{p} z=0 \quad\left(p=q^{2 \alpha}\right) .
$$

For $r \in \mathbf{C}(\operatorname{Re}(r)>0)$ we introduce the Jacobi elliptic theta function

$$
[u]_{r}=q^{\frac{u^{2}}{r}-u} \frac{\Theta_{q^{2 r}}\left(q^{2 u}\right)}{\left(q^{2 r} ; q^{2 r}\right)_{\infty}^{3}},
$$


where we have used

$$
\Theta_{p}(z)=(z ; p)_{\infty}\left(p z^{-1} ; p\right)_{\infty}(p ; p)_{\infty}, \quad(z ; p)_{\infty}=\prod_{m=0}^{\infty}\left(1-p^{m} z\right) .
$$

The Jacobi elliptic theta function satisfies the quasi-periodicity property

$$
[u+r]_{r}=-[u]_{r}, \quad[u+r \tau]_{r}=-e^{-\pi i \tau-\frac{2 \pi i}{r} u}[u]_{r},
$$

where $\tau$ such that $\operatorname{Im}(\tau)>0$ is given by $q^{2 r}=e^{-\frac{2 \pi i}{\tau}}$.

Definition 4.1 We introduce the bosonic operators $S_{i}(z)(i=1,2, \cdots, N)$ that we call the screening current as follows.

$$
\begin{aligned}
S_{i}(z) & =\frac{1}{\left(q-q^{-1}\right) z} \sum_{j=i+1}^{N}\left(S_{i}^{(j, 1)}(z)-S_{i}^{(j, 2)}(z)\right)+q S_{i}^{(N+1,0)}(z) \quad(1 \leq i \leq N-1), \\
S_{N}(z) & =-q^{-1} S_{N}^{(N+1,0)}(z) .
\end{aligned}
$$

For $2 i+1 \leq j \leq 2 N+1$ we have set

$$
S_{i}^{(j, s)}(z)=: \exp \left(-\left(\frac{1}{k+N-1} a^{i}\right)\left(z \mid \frac{k+N-1}{2}\right)\right) \widetilde{S}_{i}^{(j, s)}(z): .
$$

Here, for $1 \leq i \leq N-1$ and $i+1 \leq j \leq N$, we have set

$$
\begin{aligned}
\widetilde{S}_{i}^{(j, 1)}(z)= & : \exp \left(-b_{-}^{i, j}\left(q^{N-1-j} z\right)-(b+c)^{i, j}\left(q^{N-j} z\right)+(b+c)^{i+1, j}\left(q^{N-1-j} z\right)\right. \\
& \left.+\sum_{l=j+1}^{N}\left(b_{-}^{i+1, l}\left(q^{N-l} z\right)-b_{-}^{i, l}\left(q^{N-l-1} z\right)\right)+b_{-}^{i+1, N+1}(z)-b_{-}^{i, N+1}\left(q^{-1} z\right)\right): \\
\widetilde{S}_{i}^{(j, 2)}(z)= & : \exp \left(-b_{+}^{i, j}\left(q^{N-1-j} z\right)-(b+c)^{i, j}\left(q^{N-j-2} z\right)+(b+c)^{i+1, j}\left(q^{N-1-j} z\right)\right. \\
& \left.+\sum_{l=j+1}^{N}\left(b_{-}^{i+1, l}\left(q^{N-l} z\right)-b_{-}^{i, l}\left(q^{N-l-1} z\right)\right)+b_{-}^{i+1, N+1}(z)-b_{-}^{i, N+1}\left(q^{-1} z\right)\right): .
\end{aligned}
$$

For $1 \leq i \leq N-1$ we have set

$$
\begin{aligned}
& \widetilde{S}_{i}^{(N+1,0)}(z)=: \exp \left(b^{i, N+1}(z)+b_{+}^{i+1, N+1}(z)-b^{i+1, N+1}(q z)\right): \\
& \widetilde{S}_{N}^{(N+1,0)}(z)=: \exp \left(b^{N, N+1}(z)\right): .
\end{aligned}
$$

The $\mathbf{Z}_{2}$-grading of the screening currents are $:\left|S_{N}^{(N+1,0)}(z)\right|=1$ and zero otherwise.

Theorem 4.2 The screening currents $S_{i}(z)(i=1,2, \cdots, N)$ commute (or anti-commute) with $U_{q}(\widehat{s l}(N \mid 1))$ modulo total difference.

$$
\begin{aligned}
{\left[h_{i, m}, S_{j}(z)\right] } & =0 \\
{\left[X_{i}^{+}\left(z_{1}\right), S_{j}\left(z_{2}\right)\right] } & =0 \\
{\left[X_{i}^{-}\left(z_{1}\right), S_{j}\left(z_{2}\right)\right] } & =\frac{\delta_{i, j}}{\left(q-q^{-1}\right) z_{1}^{2}}\left(k+N-1 \partial_{z} \delta\right)\left(z_{2} / z_{1}\right) \\
& \times: \exp \left(-\left(\frac{1}{k+N-1} a^{j}\right)\left(z_{1} \mid-\frac{k+N-1}{2}\right)\right): .
\end{aligned}
$$


The screening currents $S_{i}(z)(i=1,2, \cdots, N)$ satisfy

$$
\left[u_{1}-u_{2}+\frac{A_{i, j}}{2}\right]_{k+N-1} S_{i}\left(z_{1}\right) S_{j}\left(z_{2}\right)=\left[u_{2}-u_{1}+\frac{A_{i, j}}{2}\right]_{k+N-1} S_{j}\left(z_{1}\right) S_{i}\left(z_{2}\right) .
$$

The symbol $[u]_{k+N-1}$ represents the Jacobi elliptic theta function. Here we have used $z_{j}=q^{2 u_{j}}$.

Definition 4.3 We introduce the screening operators $Q_{i}(i=1,2, \cdots, N)$ by the Jackson integral.

$$
Q_{i}=\int_{0}^{s \infty} S_{i}(z) d_{p} z, \quad\left(p=q^{2(k+N-1)}\right) .
$$

The screening operators $Q_{i}$ are convergent on the Fock space.

Corollary 4.4 The screening operators $Q_{i}(i=1,2, \cdots, N)$ commute with the quantum superalgebra $U_{q}(\widehat{s l}(N \mid 1))$.

Proposition 4.5 The screening operators $Q_{i}(i=1,2, \cdots, N)$ commute with the projection operator $\eta_{0} \xi_{0}$ of the $\xi-\eta$ system. Hence the screening operators $Q_{i}$ act on the Fock-Wakimoto module $\mathcal{F}\left(p_{a}\right)$.

\subsection{Proof}

Here we give proof of theorem 4.2 Direct calculations of the normal orderings show theorem 4.2

- Proof of (4.16) for $1 \leq i=j \leq N$.

First we show (4.16) for $1 \leq i=j \leq N-1$. The commutators vanish, $\left[X_{i}^{-(l, s)}\left(z_{1}\right), S_{i}^{(m, t)}\left(z_{2}\right)\right]=0$, for the following condition.

$$
[(l, s),(m, t)] \neq\left\{\begin{array}{cc}
{[(i, 1),(i+1,1)],[(i, 2),(i+1,2)]} & (1 \leq i \leq N-1) \\
{[(l, 1),(l+1,2)],[(l, 2),(l+1,1)]} & (1 \leq i \leq N-1, i+1 \leq l \leq N-1) \\
{[(N, 0),(N+1,0)]} & (1 \leq i \leq N-1)
\end{array}\right.
$$

Hence we have the following relations for $1 \leq i \leq N-1$.

$$
\begin{aligned}
{\left[X_{i}^{-}\left(z_{1}\right), S_{i}\left(z_{2}\right)\right]=} & q^{k+N}\left[X_{i}^{-(N, 0)}\left(z_{1}\right), S_{i}^{(N+1,0)}\left(z_{2}\right)\right] \\
& +\frac{1}{\left(q-q^{-1}\right)^{2} z_{1} z_{2}}\left\{\left[X_{i}^{-(i, 1)}\left(z_{1}\right), S_{i}^{(i+1,1)}\left(z_{2}\right)\right]+\left[X_{i}^{-(i, 2)}\left(z_{1}\right), S_{i}^{(i+1,2)}\left(z_{2}\right)\right]\right. \\
& \left.+\sum_{l=i+1}^{N-1}\left(\left[X_{i}^{-(l, 1)}\left(z_{1}\right), S_{i}^{(l+1,2)}\left(z_{2}\right)\right]+\left[X_{i}^{-(l, 2)}\left(z_{1}\right), S_{i}^{(l+1,1)}\left(z_{2}\right)\right]\right)\right\} .
\end{aligned}
$$

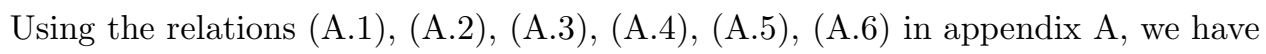

$$
\begin{aligned}
& {\left[X_{i}^{-}\left(z_{1}\right), S_{i}\left(z_{2}\right)\right]\left(q-q^{-1}\right) z_{1} z_{2} } \\
= & \delta\left(\frac{q^{-N-k-1} z_{2}}{z_{1}}\right)\left(: X_{i}^{-(N, 0)}\left(z_{1}\right) S_{i}^{(N+1,0)}\left(z_{2}\right):-: X_{i}^{-(N-1,1)}\left(z_{1}\right) S_{i}^{(N, 2)}\left(z_{2}\right):\right) \\
+ & \delta\left(\frac{q^{N-3-k-2 i} z_{2}}{z_{1}}\right)\left(: X_{i}^{-(i+1,2)}\left(z_{1}\right) S_{i}^{(i+2,1)}\left(z_{2}\right):-: X_{i}^{-(i, 2)}\left(z_{1}\right) S_{i}^{(i+1,2)}\left(z_{2}\right):\right) \\
+ & \sum_{l=i+1}^{N-2} \delta\left(\frac{q^{N-3-k-2 l} z_{2}}{z_{1}}\right)\left(: X_{i}^{-(l+1,2)}\left(z_{1}\right) S_{i}^{(l+2,1)}\left(z_{2}\right):-: X_{i}^{-(l+1,1)}\left(z_{1}\right) S_{i}^{(l+2,2)}\left(z_{2}\right):\right) \\
+ & \delta\left(\frac{q^{N+k-1} z_{2}}{z_{1}}\right): X_{i}^{-(i, 1)}\left(z_{1}\right) S_{i}^{(i+1,1)}\left(z_{2}\right):-\delta\left(\frac{q^{-N-k+1} z_{2}}{z_{1}}\right): X_{i}^{-(N, 0)}\left(z_{1}\right) S_{i}^{(N+1,0)}\left(z_{2}\right): .
\end{aligned}
$$


Using specializations (A.29), (A.31), (A.32), (A.33) in appendix A, we conclude (4.16) for $1 \leq i=j \leq$ $N-1$. Next we show (4.16) for $i=j=N$. The commutators vanish, $\left[X_{N}^{-(l, s)}\left(z_{1}\right), S_{N}^{(m, t)}\left(z_{2}\right)\right]=0$, for $[(l, s),(m, t)] \neq[(N, 1),(N+1,0)],[(N, 2),(N+1,0)]$. Hence, using the relations (A.7) and (A.8) in appendix $[$, we have

$$
\begin{aligned}
& {\left[X_{N}^{-}\left(z_{1}\right), S_{N}\left(z_{2}\right)\right]\left(q-q^{-1}\right) z_{1} z_{2} } \\
= & \delta\left(\frac{q^{N+k-1} z_{2}}{z_{1}}\right): X_{N}^{-(N, 1)}\left(z_{1}\right) S_{N}^{(N+1,0)}\left(z_{2}\right):-\delta\left(\frac{q^{N+k-1} z_{2}}{z_{1}}\right): X_{N}^{-(N, 2)}\left(z_{1}\right) S_{N}^{(N+1,0)}\left(z_{2}\right): .
\end{aligned}
$$

Using the relation (A.30) in appendix A, we have (4.16) for $i=N$. Now we have shown (4.16) for $1 \leq i=j \leq N$.

- Proof of (4.16) for $1 \leq i \neq j \leq N$.

First we show (4.16) for $i+1<j$. In this case the commutators vanish, $\left[X_{i}^{-(l, s)}\left(z_{1}\right), S_{j}^{(m, t)}\left(z_{2}\right)\right]=0$, for every $[(l, s),(m, t)]$. Hence we conclude $\left[X_{i}^{-}\left(z_{1}\right), S_{j}\left(z_{2}\right)\right]=0$. Next we show (4.16) for $j=i+1$. The commutators vanish, $\left[X_{i}^{-(l, s)}\left(z_{1}\right), S_{j}^{(m, t)}\left(z_{2}\right)\right]=0$, for the following condition.

$$
[(l, s),(m, t)] \neq[(l, 1),(l+1,2)],[(l, 2),(l+1,1)] \quad(1 \leq i \leq N-2, i+1 \leq l \leq N-1) .
$$

Hence, using the relations (A.9) and , we have the following relation for $1 \leq i \leq N-1$.

$$
\begin{aligned}
& {\left[X_{i}^{-}\left(z_{1}\right), S_{i+1}\left(z_{2}\right)\right]\left(q-q^{-1}\right) z_{1} z_{2} } \\
= & \sum_{l=i+1}^{N-1} \delta\left(\frac{q^{N-k-2 l-2} z_{2}}{z_{1}}\right)\left(-: X_{i}^{-(l, 1)}\left(z_{1}\right) S_{i+1}^{(l+1,2)}\left(z_{2}\right):+: X_{i}^{-(l, 2)}\left(z_{1}\right) S_{i+1}^{(l+1,1)}\left(z_{2}\right):\right) .
\end{aligned}
$$

Using the specialization (A.34), we conclude (4.16) for $j=i+1$. Next we show (4.16) for $1 \leq j<i \leq N-1$. The commutators vanish, $\left[X_{i}^{-(l, s)}\left(z_{1}\right), S_{j}^{(m, t)}\left(z_{2}\right)\right]=0$, for the following condition.

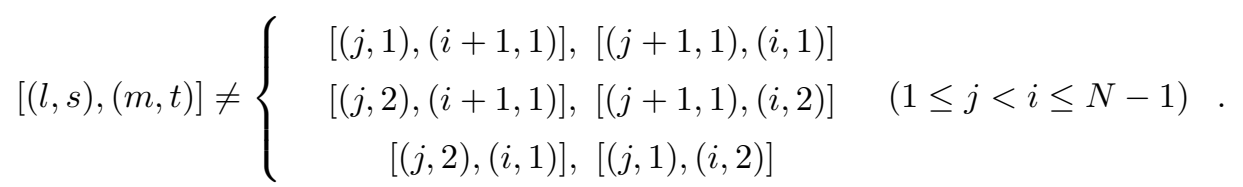

Hence, using the relations (A.11), (A.12), A.13), (A.14), (A.15), (A.16), we have the following relation for $1 \leq j<i \leq N-1$.

$$
\begin{aligned}
& {\left[X_{i}^{-}\left(z_{1}\right), S_{j}\left(z_{2}\right)\right]\left(q-q^{-1}\right) z_{1} z_{2} } \\
= & \delta\left(\frac{q^{N+k-i+j-1} z_{2}}{z_{1}}\right)\left(: X_{i}^{-(j, 1)}\left(z_{1}\right) S_{j}^{(i+1,1)}\left(z_{2}\right):-: X_{i}^{-(j+1,1)}\left(z_{1}\right) S_{j}^{(i, 1)}\left(z_{2}\right):\right. \\
& -: X_{i}^{-(j, 2)}\left(z_{1}\right) S_{j}^{(i+1,1)}\left(z_{2}\right):+: X_{i}^{-(j+1,1)}\left(z_{1}\right) S_{j}^{(i, 2)}\left(z_{2}\right): \\
& \left.-q^{-1}: X_{i}^{-(j, 2)}\left(z_{1}\right) S_{j}^{(i, 1)}\left(z_{2}\right):+q^{-1}: X_{i}^{-(j, 1)}\left(z_{1}\right) S_{j}^{(i, 2)}\left(z_{2}\right):\right) .
\end{aligned}
$$

Using the specializations (A.35), (A.36), (A.37), we have $\left[X_{i}^{-}\left(z_{1}\right), S_{j}\left(z_{2}\right)\right]=0$ for $1 \leq j<i \leq N-1$. Next we show (4.16) for $1 \leq j \leq N-1$ and $i=N$. The commutators vanish, $\left[X_{i}^{-(l, s)}\left(z_{1}\right), S_{j}^{(m, t)}\left(z_{2}\right)\right]=0$, for the following condition.

$$
[(l, s),(m, t)] \neq\left\{\begin{array}{c}
{[(j, 1),(N, 2)],[(j, 2),(N, 1)]} \\
{[(j, 1),(N+1,0)],[(j+1,1),(N, 1)] \quad(1 \leq j \leq N-1) .} \\
{[(j, 2),(N+1,0)],[(j+1,1),(N, 2)]}
\end{array}\right.
$$


Hence, using the relations (A.12), (A.14), (A.15), (A.16), (A.17), (A.18) we have the following relation for $1 \leq j \leq N-1$.

$$
\begin{aligned}
& {\left[X_{N}^{-}\left(z_{1}\right), S_{j}\left(z_{2}\right)\right]\left(q-q^{-1}\right) q^{N-j-2} z_{1} z_{2} } \\
= & \delta\left(\frac{q^{k+j-1} z_{2}}{z_{1}}\right)\left(q^{-2}: X_{N}^{-(j, 1)}\left(z_{1}\right) S_{j}^{(N, 2)}\left(z_{2}\right):-q^{-2}: X_{N}^{-(j, 2)}\left(z_{1}\right) S_{j}^{(N, 1)}\left(z_{2}\right):\right. \\
& -: X_{N}^{-(j, 1)}\left(z_{1}\right) S_{j}^{(N+1,0)}\left(z_{2}\right):+: X_{N}^{-(j+1,1)}\left(z_{1}\right) S_{j}^{(N, 1)}\left(z_{2}\right): \\
& \left.+-: X_{N}^{-(j, 2)}\left(z_{1}\right) S_{j}^{(N+1,0)}\left(z_{2}\right):+: X_{N}^{-(j+1,1)}\left(z_{1}\right) S_{j}^{(N, 2)}\left(z_{2}\right):\right) .
\end{aligned}
$$

Using the specializations (A.37), A.38), A.39, we have $\left[X_{N}^{-}\left(z_{1}\right), S_{j}\left(z_{2}\right)\right]=0$ for $1 \leq j \leq N-1$. Now we have shown $\left[X_{i}^{-}\left(z_{1}\right), S_{j}\left(z_{2}\right)\right]=0$ for $1 \leq i \neq j \leq N$.

- Proof of (4.15) for $1 \leq i=j \leq N$.

The commutators vanish, $\left[X_{i}^{+(l, s)}\left(z_{1}\right), S_{i}^{(m, t)}\left(z_{2}\right)\right]=0$, for the condition

$$
[(l, s),(m, t)] \neq[(i, 1),(i+1,2)],[(i, 2),(i+1,1)] \quad(1 \leq i \leq N-1) .
$$

We have $\left[X_{N}^{+}\left(z_{1}\right), S_{N}\left(z_{2}\right)\right]=0$. For $1 \leq i \leq N-1$, using the relations (A.19) and (A.20), we have

$$
\begin{aligned}
& {\left[X_{i}^{+}\left(z_{1}\right), S_{i}\left(z_{2}\right)\right]\left(q-q^{-1}\right) z_{1} z_{2} } \\
= & \delta\left(\frac{q^{N-2 i-1} z_{2}}{z_{1}}\right)\left(-: X_{i}^{+(i, 1)}\left(z_{1}\right) S_{i}^{(i+1,2)}\left(z_{2}\right):+: X_{i}^{+(i, 2)}\left(z_{1}\right) S_{i}^{(i+1,1)}\left(z_{2}\right):\right) .
\end{aligned}
$$

Using the specialization (A.40), we conclude $\left[X_{i}^{+}\left(z_{1}\right), S_{i}\left(z_{2}\right)\right]=0$ for $1 \leq i \leq N-1$.

- Proof of (4.15) for $1 \leq i \neq j \leq N$.

First we show (4.15) for $1 \leq i \leq N-1,1 \leq j \leq i-1$. The commutators vanish, $\left[X_{i}^{+(l, s)}\left(z_{1}\right), S_{j}^{(m, t)}\left(z_{2}\right)\right]=$ 0 , for the following condition.

$$
[(l, s),(m, t)] \neq\left\{\begin{array}{l}
{[(j, 1),(i, 2)],[(j+1,2),(i+1,1)]} \\
{[(j, 1),(i+1,2)],[(j, 2),(i+1,1)]} \\
{[(j, 2),(i, 2)],[(j+1,2),(i+1,2)]}
\end{array} \quad(1 \leq i \leq N-1,1 \leq j \leq i-1)\right.
$$

Hence, using the relations (A.21), (A.22), A.25), (A.26), (A.27), we have the following relation for $1 \leq i \leq N-1$ and $1 \leq j \leq i-1$.

$$
\begin{aligned}
& {\left[X_{i}^{+}\left(z_{1}\right), S_{j}\left(z_{2}\right)\right]\left(q-q^{-1}\right) z_{1} z_{2} } \\
= & \delta\left(\frac{q^{N-i-j-1} z_{2}}{z_{1}}\right)\left(q: X_{i}^{+(j, 1)}\left(z_{1}\right) S_{j}^{(i, 2)}\left(z_{2}\right):-q: X_{i}^{+(j+1,2)}\left(z_{1}\right) S_{j}^{(i+1,1)}\left(z_{2}\right):\right. \\
& -: X_{i}^{+(j, 1)}\left(z_{1}\right) S_{j}^{(i+1,2)}\left(z_{2}\right):+: X_{i}^{+(j, 2)}\left(z_{1}\right) S_{j}^{(i+1,1)}\left(z_{2}\right): \\
& \left.-q: X_{i}^{+(j, 2)}\left(z_{1}\right) S_{j}^{(i, 2)}\left(z_{2}\right):+q: X_{i}^{+(j+1,2)}\left(z_{1}\right) S_{j}^{(i+1,2)}\left(z_{2}\right):\right) .
\end{aligned}
$$

Using the specializations (A.41), A.43), A.44), we conclude $\left[X_{i}^{+}\left(z_{1}\right), S_{j}\left(z_{2}\right)\right]=0$ for $1 \leq i \leq N-$ $1,1 \leq j \leq i-1$. Next we show (4.15) for $i=N$ and $1 \leq j \leq N-1$. The commutators vanish, $\left[X_{i}^{+(l, s)}\left(z_{1}\right), S_{j}^{(m, t)}\left(z_{2}\right)\right]=0$, for the following condition.

$$
[(l, s),(m, t)] \neq[(j, 0),(N, 2)],[(j+1,0),(N+1,0)] \quad(1 \leq j \leq N-1) .
$$


Hence, using the relations (A.23) and (A.24), we have

$$
\begin{aligned}
& {\left[X_{N}^{+}\left(z_{1}\right), S_{j}\left(z_{2}\right)\right] q^{-N+1} z_{2} } \\
= & \delta\left(\frac{q^{-j-1} z_{2}}{z_{1}}\right)\left(: X_{N}^{+(j, 0)}\left(z_{1}\right) S_{j}^{(N, 2)}\left(z_{2}\right):-: X_{N}^{+(j+1,0)}\left(z_{1}\right) S_{j}^{(N+1,0)}\left(z_{2}\right):\right) .
\end{aligned}
$$

Using the specialization (A.42), we conclude $\left[X_{N}^{+}\left(z_{1}\right), S_{j}\left(z_{2}\right)\right]=0$ for $1 \leq j \leq N-1$. Now we have shown the commutation relation (4.15).

- Proof of (4.14). This is a direct consequence of the relation,

$$
\left[\Psi_{i}^{ \pm}\left(z_{1}\right), S_{j}^{(l, s)}\left(z_{2}\right)\right]=0
$$

for every $i, j$, and $(l, s)$.

- Proof of (4.17). This is a direct consequence of the relation,

$$
\left[u_{1}-u_{2}+\frac{A_{i, j}}{2}\right]_{k+N-1} S_{i}^{(l, s)}\left(z_{1}\right) S_{j}^{(m, t)}\left(z_{2}\right)=\left[u_{2}-u_{1}+\frac{A_{i, j}}{2}\right]_{k+N-1} S_{j}^{(m, t)}\left(z_{2}\right) S_{i}^{(l, s)}\left(z_{1}\right),
$$

for every $(i, j)$ and $[(l, s),(m, t)]$.

\section{$5 \quad$ Vertex operator}

In this section we propose bosonizations of the vertex operators for the quantum superalgebra $U_{q}(\widehat{s l}(N \mid 1))$ 30. We check that the vertex operators are the intertwiners among the Fock-Wakimoto module and the typical representation for small rank $N \leq 4$.

\subsection{Level-zero representation}

We discuss level-zero representation of $U_{q}(\widehat{s l}(3 \mid 1))$ in this section (resp. $U_{q}(\widehat{s l}(4 \mid 1))$ in appendix $\mathbb{B}$ ), that we will use for the investigation of the vertex operator. Let $V_{\alpha}$ be the one parameter family of the $2^{N}$-dimensional typical representation of $U_{q}(\operatorname{sl}(N \mid 1))$ [36, 37]. In the case of $U_{q}(\operatorname{sl}(3 \mid 1))$, we choose the basis $\left\{v_{j}\right\}_{1 \leq j \leq 8}$ of $V_{\alpha}$ and assign them the $\mathbf{Z}_{2}$-gradings as following.

$$
\left|v_{1}\right|=\left|v_{5}\right|=\left|v_{6}\right|=\left|v_{7}\right|=0, \quad\left|v_{2}\right|=\left|v_{3}\right|=\left|v_{4}\right|=\left|v_{8}\right|=1 .
$$

In the homogeneous gradation, the evaluation representation $V_{\alpha, z}$ of $U_{q}(\widehat{s l}(3 \mid 1))$ is given by

$$
\begin{aligned}
h_{1} & =E_{3,3}-E_{4,4}+E_{5,5}-E_{6,6}, \\
h_{2} & =E_{2,2}-E_{3,3}+E_{6,6}-E_{7,7}, \\
h_{3} & =\alpha\left(E_{1,1}+E_{2,2}\right)+(\alpha+1)\left(E_{3,3}+E_{4,4}+E_{5,5}+E_{6,6}\right)+(\alpha+2)\left(E_{7,7}+E_{8,8}\right), \\
e_{1} & =E_{3,4}+E_{5,6}, \\
e_{2} & =E_{2,3}+E_{6,7}, \\
e_{3} & =\sqrt{[\alpha]} E_{1,2}-\sqrt{[\alpha+1]}\left(E_{3,5}+E_{4,6}\right)+\sqrt{[\alpha+2]} E_{7,8}, \\
f_{1} & =E_{4,3}+E_{6,5},
\end{aligned}
$$




$$
\begin{aligned}
& f_{2}=E_{3,2}+E_{7,6}, \\
& f_{3}=\sqrt{[\alpha]} E_{2,1}-\sqrt{[\alpha+1]}\left(E_{5,3}+E_{6,4}\right)+\sqrt{[\alpha+2]} E_{8,7}, \\
& h_{0}=-\alpha\left(E_{1,1}+E_{4,4}\right)-(\alpha+1)\left(E_{2,2}+E_{3,3}+E_{6,6}+E_{7,7}\right)-(\alpha+2)\left(E_{5,5}+E_{8,8}\right), \\
& e_{0}=-z\left(\sqrt{[\alpha]} E_{4,1}-\sqrt{[\alpha+1]}\left(E_{6,2}+E_{7,3}\right)+\sqrt{[\alpha+2]} E_{8,5}\right), \\
& f_{0}=z^{-1}\left(\sqrt{[\alpha]} E_{1,4}-\sqrt{[\alpha+1]}\left(E_{2,6}+E_{3,7}\right)+\sqrt{[\alpha+2]} E_{5,8}\right) .
\end{aligned}
$$

We set the dual representation $V_{\alpha, z}^{* S}$ of $U_{q}(\widehat{s l}(3 \mid 1))$ by

$$
\pi_{V_{\alpha, z}^{*,}}(a)=\left(\pi_{V_{\alpha, z}}(S(a))\right)^{s t} \quad \text { for } \quad a \in U_{q}(\widehat{s l}(3 \mid 1)),
$$

where we have used the antipode $S$ and have introduced the supertransposition "st" by

$$
\left(E_{i, j}\right)^{s t}=(-1)^{\left|v_{i}\right|\left(\left|v_{i}\right|+\left|v_{j}\right|\right)} E_{j, i}
$$

We have chosen the dual basis $\left\{v_{j}^{*}\right\}_{1 \leq j \leq 8}$ of $V_{\alpha}^{* S}$ and assign them the $\mathbf{Z}_{2}$-gradings as following.

$$
\left|v_{1}^{*}\right|=\left|v_{5}^{*}\right|=\left|v_{6}^{*}\right|=\left|v_{7}^{*}\right|=0, \quad\left|v_{2}^{*}\right|=\left|v_{3}^{*}\right|=\left|v_{4}^{*}\right|=\left|v_{8}^{*}\right|=1 .
$$

In the homogeneous gradation, the evaluation representation $V_{\alpha, z}^{* S}$ of $U_{q}(\widehat{s l}(3 \mid 1))$ is given by

$$
\begin{aligned}
& h_{1}=-E_{3,3}+E_{4,4}-E_{5,5}+E_{6,6}, \\
& h_{2}=-E_{2,2}+E_{3,3}-E_{6,6}+E_{7,7}, \\
& h_{3}=-\alpha\left(E_{1,1}+E_{2,2}\right)-(\alpha+1)\left(E_{3,3}+E_{4,4}+E_{5,5}+E_{6,6}\right)-(\alpha+2)\left(E_{7,7}+E_{8,8}\right), \\
& e_{1}=-q^{-1}\left(E_{4,3}+E_{6,5}\right) \\
& e_{2}=-q^{-1}\left(E_{3,2}+E_{7,6}\right) \\
& e_{3}=-\left(\sqrt{[\alpha]} q^{-\alpha} E_{2,1}+\sqrt{[\alpha+1]} q^{-\alpha-1}\left(E_{5,3}+E_{6,4}\right)+\sqrt{[\alpha+2]} q^{-\alpha-2} E_{8,7}\right), \\
& f_{1}=-q\left(E_{3,4}+E_{5,6}\right) \\
& f_{2}=-q\left(E_{2,3}+E_{6,7}\right) \\
& f_{3}=\sqrt{[\alpha]} q^{\alpha} E_{1,2}+\sqrt{[\alpha+1]} q^{\alpha+1}\left(E_{3,5}+E_{4,6}\right)+\sqrt{[\alpha+2]} q^{\alpha+2} E_{7,8}, \\
& h_{0}=\alpha\left(E_{1,1}+E_{4,4}\right)+(\alpha+1)\left(E_{2,2}+E_{3,3}+E_{6,6}+E_{7,7}\right)+(\alpha+2)\left(E_{5,5}+E_{8,8}\right), \\
& e_{0}=-z\left(\sqrt{[\alpha]} q^{\alpha} E_{1,4}+\sqrt{[\alpha+1]} q^{\alpha+1}\left(E_{2,6}+E_{3,7}\right)+\sqrt{[\alpha+2]} q^{\alpha+2} E_{5,8}\right), \\
& f_{0}=-z^{-1}\left(\sqrt{[\alpha]} q^{-\alpha} E_{4,1}+\sqrt{[\alpha+1]} q^{-\alpha-1}\left(E_{6,2}+E_{7,3}\right)+\sqrt{[\alpha+2]} q^{-\alpha-2} E_{8,5}\right) .
\end{aligned}
$$

We give the level-zero realization of the Drinfeld generators.

Proposition 5.1 On $V_{\alpha, z}$, the Drinfeld generators of $U_{q}(\widehat{s l}(3 \mid 1))$ are realized by

$$
\begin{aligned}
h_{1, m} & =\frac{[m]}{m}\left(q^{\alpha+2} z\right)^{m}\left(q^{-m} E_{3,3}-q^{m} E_{4,4}+q^{-m} E_{5,5}-q^{m} E_{6,6}\right), \\
h_{2, m} & =\frac{[m]}{m}\left(q^{\alpha+2} z\right)^{m}\left(q^{-2 m} E_{2,2}-E_{3,3}+E_{6,6}-q^{2 m} E_{7,7}\right), \\
h_{3, m} & =\frac{1}{m} z^{m}\left([\alpha m]\left(E_{1,1}+E_{2,2}\right)+[(\alpha+1) m] q^{m}\left(E_{3,3}+E_{4,4}+E_{5,5}+E_{6,6}\right)\right.
\end{aligned}
$$




$$
\begin{aligned}
& \left.+[(\alpha+2) m] q^{2 m}\left(E_{7,7}+E_{8,8}\right)\right), \\
x_{1, n}^{+}= & \left(q^{\alpha+2} z\right)^{n}\left(E_{3,4}+E_{5,6}\right), \\
x_{2, n}^{+}= & \left(q^{\alpha+2} z\right)^{n}\left(q^{-n} E_{2,3}+q^{n} E_{6,7}\right), \\
x_{3, n}^{+}= & \left(q^{\alpha+2} z\right)^{n}\left(\sqrt{[\alpha]} q^{-2 n} E_{1,2}-\sqrt{[\alpha+1]}\left(E_{3,5}+E_{4,6}\right)+\sqrt{[\alpha+2]} q^{2 n} E_{7,8}\right), \\
x_{1, n}^{-}= & \left(q^{\alpha+2} z\right)^{n}\left(E_{4,3}+E_{6,5}\right), \\
x_{2, n}^{-}= & \left(q^{\alpha+2} z\right)^{n}\left(q^{-n} E_{3,2}+q^{n} E_{7,6}\right), \\
x_{3, n}^{-}= & \left(q^{\alpha+2} z\right)^{n}\left(\sqrt{[\alpha]} q^{-2 n} E_{2,1}-\sqrt{[\alpha+1]}\left(E_{5,3}+E_{6,4}\right)+\sqrt{[\alpha+2]} q^{2 n} E_{8,7}\right) .
\end{aligned}
$$

On $V_{\alpha, z}^{* S}$, the Drinfeld generators of $U_{q}(\widehat{s l}(3 \mid 1))$ are realized by

$$
\begin{aligned}
h_{1, m}= & \frac{[m]_{q}}{m}\left(q^{-\alpha-2} z\right)^{m}\left(-q^{m} E_{3,3}+q^{-m} E_{4,4}-q^{m} E_{5,5}+q^{-m} E_{6,6}\right), \\
h_{2, m}= & \frac{[m]_{q}}{m}\left(q^{-\alpha-2} z\right)^{m}\left(-q^{2 m} E_{2,2}+E_{3,3}-E_{6,6}+q^{-2 m} E_{7,7}\right), \\
h_{3, m}= & \frac{-1}{m} z^{m}\left([\alpha m]\left(E_{1,1}+E_{2,2}\right)+[(\alpha+1) m] q^{-m}\left(E_{3,3}+E_{4,4}+E_{5,5}+E_{6,6}\right)\right. \\
& \left.+[(\alpha+2) m] q^{-2 m}\left(E_{7,7}+E_{8,8}\right)\right), \\
x_{1, n}^{+}= & -q^{-1}\left(q^{-\alpha-2} z\right)^{n}\left(E_{4,3}+E_{6,5}\right), \\
x_{2, n}^{+}= & -q^{-1}\left(q^{-\alpha-2} z\right)^{n}\left(q^{n} E_{3,2}+q^{-n} E_{7,6}\right), \\
x_{3, n}^{+}= & -\left(q^{-\alpha-2} z\right)^{n}\left(\sqrt{[\alpha]} q^{-\alpha+2 n} E_{2,1}+\sqrt{[\alpha+1]} q^{-\alpha-1}\left(E_{5,3}+E_{6,4}\right)\right. \\
& \left.+\sqrt{[\alpha+2]} q^{-\alpha-2-2 n} E_{8,7}\right), \\
x_{1, n}^{-}= & -q\left(q^{-\alpha} z\right)^{n}\left(E_{3,4}+E_{5,6}\right), \\
x_{2, n}^{-}= & -q\left(q^{-\alpha-2} z\right)^{n}\left(q^{n} E_{2,3}+q^{-n} E_{6,7}\right), \\
x_{3, n}^{-}= & \left(q^{-\alpha-2} z\right)^{n}\left(\sqrt{[\alpha]} q^{\alpha+2 n} E_{1,2}+\sqrt{[\alpha+1]} q^{\alpha+1}\left(E_{3,5}+E_{4,6}\right)\right. \\
& \left.+\sqrt{[\alpha+2]} q^{\alpha+2-2 n} E_{7,8}\right) .
\end{aligned}
$$

In appendix [B] we summarize the case of $U_{q}(\widehat{s l}(4 \mid 1))$. The case of $U_{q}(\widehat{s l}(2 \mid 1))$ is summarized in [11].

\subsection{Vertex operator}

Let $\mathcal{F}$ and $\mathcal{F}^{\prime}$ be level $k$ highest weight $U_{q}(\widehat{s l}(N \mid 1))$-modules. Let $V_{\alpha}$ and $V_{\alpha}^{* S}$ be $2^{N}$-dimensional typical representation with a parameters $\alpha$ 37. The representations $V_{\alpha}$ and $V_{\alpha}^{* S}$ are irreducible if and only if $\alpha \neq 0,-1,-2, \cdots,-N+1$. Let $V_{\alpha, z}$ and $V_{\alpha, z}^{* S}$ be the evaluation module and its dual of the typical representation. Consider the following intertwiners of $U_{q}(\widehat{s l}(N \mid 1))$-module [30].

$$
\Phi(z): \mathcal{F} \longrightarrow \mathcal{F}^{\prime} \otimes V_{\alpha, z}, \quad \Phi^{*}(z): \mathcal{F} \longrightarrow \mathcal{F}^{\prime} \otimes V_{\alpha, z}^{* S}
$$

They are intertwiners in the sense that for any $x \in U_{q}(\widehat{s l}(N \mid 1))$,

$$
\Phi(z) \cdot x=\Delta(x) \cdot \Phi(z), \quad \Phi^{*}(z) \cdot x=\Delta(x) \cdot \Phi^{*}(z) .
$$


We expand the intertwining operators.

$$
\Phi(z)=\sum_{j=1}^{2^{N}} \Phi_{j}(z) \otimes v_{j}, \quad \Phi^{*}(z)=\sum_{j=1}^{2^{N}} \Phi_{j}^{*}(z) \otimes v_{j}^{*} .
$$

We set the $\mathbf{Z}_{2}$-grading of the intertwiner be $|\Phi(z)|=\left|\Phi^{*}(z)\right|=0$. In what follow we focus our attention on $\operatorname{rank} N \leq 4$ case.

Proposition 5.2 For $\alpha \neq 0,-1,-2$, the operator $\Phi(z)$ for $U_{q}(\widehat{s l}(3 \mid 1))$ is determined by the component $\Phi_{8}(z)$. More explicitly, we have

$$
\begin{aligned}
\Phi_{3}(z) & =\left[\Phi_{4}(z), f_{1}\right]_{q}, \quad \Phi_{5}(z)=\left[\Phi_{6}(z), f_{1}\right]_{q}, \\
\Phi_{2}(z) & =\left[\Phi_{3}(z), f_{2}\right]_{q}, \quad \Phi_{6}(z)=\left[\Phi_{7}(z), f_{2}\right]_{q}, \\
\Phi_{1}(z) & =\frac{1}{\sqrt{[\alpha]}}\left[\Phi_{2}(z), f_{3}\right]_{q^{-\alpha}}, \quad \Phi_{3}(z)=\frac{-1}{\sqrt{[\alpha+1]}}\left[\Phi_{5}(z), f_{3}\right]_{q^{-\alpha-1}}, \\
\Phi_{4}(z) & =\frac{-1}{\sqrt{[\alpha+1]}}\left[\Phi_{6}(z), f_{3}\right]_{q^{-\alpha-1}}, \quad \Phi_{7}(z)=\frac{1}{\sqrt{[\alpha+2]}}\left[\Phi_{8}(z), f_{3}\right]_{q^{-\alpha-2}} .
\end{aligned}
$$

For $\alpha \neq 0,-1,-2$, the operator $\Phi^{*}(z)$ for $U_{q}(\widehat{s l}(3 \mid 1))$ is determined by the component $\Phi_{1}^{*}(z)$. More explicitly, we have

$$
\begin{aligned}
\Phi_{4}^{*}(z) & =\left[f_{1}, \Phi_{3}^{*}(z)\right]_{q^{-1}}, \quad \Phi_{6}^{*}(z)=\left[f_{1}, \Phi_{5}^{*}(z)\right]_{q^{-1}}, \\
\Phi_{3}^{*}(z) & =\left[f_{2}, \Phi_{2}^{*}(z)\right]_{q^{-1}}, \quad \Phi_{7}^{*}(z)=\left[f_{2}, \Phi_{6}^{*}(z)\right]_{q^{-1}}, \\
\Phi_{2}^{*}(z) & =\frac{1}{\sqrt{[\alpha]}}\left[f_{3}, \Phi_{1}^{*}(z)\right]_{q^{-\alpha}}, \quad \Phi_{5}^{*}(z)=\frac{-1}{\sqrt{[\alpha+1]}}\left[f_{3}, \Phi_{3}^{*}(z)\right]_{q^{-\alpha-1}}, \\
\Phi_{6}^{*}(z) & =\frac{-1}{\sqrt{[\alpha+1]}}\left[f_{3}, \Phi_{4}^{*}(z)\right]_{q^{-\alpha-1}}, \quad \Phi_{8}^{*}(z)=\frac{1}{\sqrt{[\alpha+2]}}\left[f_{3}, \Phi_{7}^{*}(z)\right]_{q^{-\alpha-2}} .
\end{aligned}
$$

Proposition 5.3 For $\alpha \neq 0,-1,-2,-3$, the operator $\Phi(z)$ for $U_{q}(\widehat{s l}(4 \mid 1))$ is determined by the component $\Phi_{16}(z)$. More explicitly, we have

$$
\begin{aligned}
& \Phi_{4}(z)=\left[\Phi_{6}(z), f_{1}\right]_{q}, \quad \Phi_{7}(z)=\left[\Phi_{8}(z), f_{1}\right]_{q}, \\
& \Phi_{9}(z)=\left[\Phi_{10}(z), f_{1}\right]_{q}, \quad \Phi_{11}(z)=\left[\Phi_{13}(z), f_{1}\right]_{q}, \\
& \Phi_{3}(z)=\left[\Phi_{4}(z), f_{2}\right]_{q}, \quad \Phi_{5}(z)=\left[\Phi_{7}(z), f_{2}\right]_{q}, \\
& \Phi_{9}(z)=\left[\Phi_{10}(z), f_{2}\right]_{q}, \quad \Phi_{11}(z)=\left[\Phi_{13}(z), f_{2}\right]_{q}, \\
& \Phi_{2}(z)=\left[\Phi_{3}(z), f_{3}\right]_{q}, \quad \Phi_{7}(z)=\left[\Phi_{9}(z), f_{3}\right]_{q}, \\
& \Phi_{8}(z)=\left[\Phi_{10}(z), f_{3}\right]_{q}, \quad \Phi_{14}(z)=\left[\Phi_{15}(z), f_{2}\right]_{q}, \\
& \Phi_{1}(z)=\frac{-1}{\sqrt{[\alpha]}}\left[\Phi_{2}(z), f_{4}\right]_{q^{-\alpha}}, \quad \Phi_{3}(z)=\frac{-1}{\sqrt{[\alpha+1]}}\left[\Phi_{5}(z), f_{4}\right]_{q^{-\alpha-1}}, \\
& \Phi_{4}(z)=\frac{-1}{\sqrt{[\alpha+1]}}\left[\Phi_{7}(z), f_{4}\right]_{q^{-\alpha-1}}, \quad \Phi_{6}(z)=\frac{-1}{\sqrt{[\alpha+1]}}\left[\Phi_{8}(z), f_{4}\right]_{q^{-\alpha-1}}, \\
& \Phi_{9}(z)=\frac{-1}{\sqrt{[\alpha+2]}}\left[\Phi_{11}(z), f_{4}\right]_{q^{-\alpha-2}}, \quad \Phi_{10}(z)=\frac{-1}{\sqrt{[\alpha+2]}}\left[\Phi_{13}(z), f_{4}\right]_{q^{-\alpha-2}}, \\
& \Phi_{12}(z)=\frac{-1}{\sqrt{[\alpha+2]}}\left[\Phi_{14}(z), f_{4}\right]_{q^{-\alpha-2}}, \quad \Phi_{15}(z)=\frac{-1}{\sqrt{[\alpha+3]}}\left[\Phi_{16}(z), f_{4}\right]_{q^{-\alpha-3}} .
\end{aligned}
$$


For $\alpha \neq 0,-1,-2,-3$, the operator $\Phi^{*}(z)$ for $U_{q}(\widehat{s l}(4 \mid 1))$ is determined by the component $\Phi_{1}^{*}(z)$. More explicitly, we have

$$
\begin{aligned}
& \Phi_{6}^{*}(z)=\left[f_{1}, \Phi_{4}^{*}(z)\right]_{q^{-1}}, \quad \Phi_{8}^{*}(z)=\left[f_{1}, \Phi_{7}^{*}(z)\right]_{q^{-1}}, \\
& \Phi_{10}^{*}(z)=\left[f_{1}, \Phi_{9}^{*}(z)\right]_{q^{-1}}, \quad \Phi_{13}^{*}(z)=\left[f_{1}, \Phi_{11}^{*}(z)\right]_{q^{-1}}, \\
& \Phi_{4}^{*}(z)=\left[f_{2}, \Phi_{3}^{*}(z)\right]_{q^{-1}}, \quad \Phi_{7}^{*}(z)=\left[f_{2}, \Phi_{5}^{*}(z)\right]_{q^{-1}}, \\
& \Phi_{10}^{*}(z)=\left[f_{2}, \Phi_{9}^{*}(z)\right]_{q^{-1}}, \quad \Phi_{13}^{*}(z)=\left[f_{2}, \Phi_{11}^{*}(z)\right]_{q^{-1}}, \\
& \Phi_{3}^{*}(z)=\left[f_{3}, \Phi_{2}^{*}(z)\right]_{q^{-1}}, \quad \Phi_{9}^{*}(z)=\left[f_{3}, \Phi_{7}^{*}(z)\right]_{q^{-1}}, \\
& \Phi_{10}^{*}(z)=\left[f_{3}, \Phi_{8}^{*}(z)\right]_{q^{-1}}, \quad \Phi_{15}^{*}(z)=\left[f_{3}, \Phi_{14}^{*}(z)\right]_{q^{-1}}, \\
& \Phi_{2}^{*}(z)=\frac{-1}{\sqrt{[\alpha]}}\left[f_{4}, \Phi_{1}^{*}(z)\right]_{q^{-\alpha}}, \quad \Phi_{5}^{*}(z)=\frac{1}{\sqrt{[\alpha+1]}}\left[f_{4}, \Phi_{3}^{*}(z)\right]_{q^{-\alpha-1}}, \\
& \Phi_{7}^{*}(z)=\frac{1}{\sqrt{[\alpha+1]}}\left[f_{4}, \Phi_{4}^{*}(z)\right]_{q^{-\alpha-1}}, \quad \Phi_{8}^{*}(z)=\frac{1}{\sqrt{[\alpha+1]}}\left[f_{4}, \Phi_{6}^{*}(z)\right]_{q^{-\alpha-1}}, \\
& \Phi_{11}^{*}(z)=\frac{-1}{\sqrt{[\alpha+2]}}\left[f_{4}, \Phi_{9}^{*}(z)\right]_{q^{-\alpha-2}}, \quad \Phi_{13}^{*}(z)=\frac{-1}{\sqrt{[\alpha+2]}}\left[f_{4}, \Phi_{10}^{*}(z)\right]_{q^{-\alpha-2}}, \\
& \Phi_{14}^{*}(z)=\frac{-1}{\sqrt{[\alpha+2]}}\left[f_{4}, \Phi_{12}^{*}(z)\right]_{q^{-\alpha-2}}, \quad \Phi_{16}^{*}(z)=\frac{-1}{\sqrt{[\alpha+3]}}\left[f_{4}, \Phi_{15}^{*}(z)\right]_{q^{-\alpha-3}} .
\end{aligned}
$$

The case of $U_{q}(\widehat{s l}(2 \mid 1))$ is summarized in [11. Next we determine the relations between the components $\Phi_{2^{N}}(z), \Phi_{1}^{*}(z)$ and the Drinfeld generators. We use the coproduct

$$
\begin{aligned}
& \Delta\left(h_{i}\right)=h_{i} \otimes 1+1 \otimes h_{i}, \\
& \Delta\left(h_{i, m}\right)=h_{i, m} \otimes q^{\frac{c m}{2}}+q^{\frac{3 c m}{2}} \otimes h_{i, m} \quad(m>0), \\
& \Delta\left(h_{i,-m}\right)=h_{i,-m} \otimes q^{-\frac{3 c m}{2}}+q^{-\frac{c m}{2}} \otimes h_{i,-m} \quad(m>0) .
\end{aligned}
$$

Proposition 5.4 The component $\Phi_{2^{N}}(z)$ associated with $U_{q}(\widehat{s l}(N \mid 1))$ satisfy

$$
\begin{aligned}
& {\left[h_{i}, \Phi_{2^{N}}(z)\right]=-\delta_{i, N}(\alpha+N-1) \Phi_{2^{N}}(z) \quad(1 \leq i \leq N),} \\
& {\left[h_{i, m}, \Phi_{2^{N}}(z)\right]=-\delta_{i, N} q^{\left(N-1+\frac{3 k}{2}\right) m} \frac{[(\alpha+N-1) m]}{m} z^{m} \Phi_{2^{N}}(z) \quad(m>0,1 \leq i \leq N),} \\
& {\left[h_{i,-m}, \Phi_{2^{N}}(z)\right]=-\delta_{i, N} q^{\left(-N+1-\frac{k}{2}\right) m} \frac{[(\alpha+N-1) m]}{m} z^{-m} \Phi_{2^{N}}(z) \quad(m>0,1 \leq i \leq N),} \\
& {\left[X_{i}^{+}\left(z_{1}\right), \Phi_{2^{N}}\left(z_{2}\right)\right]=0 \quad(1 \leq i \leq N) .}
\end{aligned}
$$

The component $\Phi_{1}^{*}(z)$ associated with $U_{q}(\widehat{s l}(N \mid 1))$ satisfy

$$
\begin{aligned}
& {\left[h_{i}, \Phi_{1}^{*}(z)\right]=\delta_{i, N} \alpha \Phi_{1}^{*}(z) \quad(1 \leq i \leq N),} \\
& {\left[h_{i, m}, \Phi_{1}^{*}(z)\right]=\delta_{i, N} q^{\frac{3 k}{2} m} \frac{[\alpha m]}{m} z^{m} \Phi_{1}^{*}(z) \quad(m>0,1 \leq i \leq N),} \\
& {\left[h_{i,-m}, \Phi_{1}^{*}(z)\right]=\delta_{i, N} q^{-\frac{k}{2} m} \frac{[\alpha m]}{m} z^{-m} \Phi_{1}^{*}(z) \quad(m>0,1 \leq i \leq N),} \\
& {\left[X_{i}^{+}\left(z_{1}\right), \Phi_{1}^{*}\left(z_{2}\right)\right]=0 \quad(1 \leq i \leq N) .}
\end{aligned}
$$

We have checked this proposition for rank $N=2,3,4$.

In order to construct bosonizations of $\Phi_{2^{N}}(z)$ and $\Phi_{1}^{*}(z)$, we introduce a bosonic operator $\phi^{l_{a}}(z \mid \beta)$. 
Definition 5.5 For $l_{a}=\left(l_{a}^{1}, l_{a}^{2}, \cdots, l_{a}^{N}\right) \in \mathbf{C}^{N}$ and $\beta \in \mathbf{C}$, we set the bosonic operator $\phi^{l_{a}}(z \mid \beta)$ by

$$
\phi^{l_{a}}(z \mid \beta)=: \exp \left(\sum_{i, j=1}^{N}\left(\frac{l_{a}^{i}}{k+N-1} \frac{\operatorname{Min}(i, j)}{N-1} \frac{N-1-\operatorname{Max}(i, j)}{1} a^{j}\right)(z \mid \beta)\right): .
$$

We call the operator $\phi^{l_{a}}(z \mid \beta)$ the "elementary vertex operator".

Proposition 5.6 The highest vector $|\lambda\rangle=\left|l_{a}, 0,0\right\rangle$ of $U_{q}(\widehat{s l}(N \mid 1))$ is created from the Fock vacuum $|0\rangle$ and $\phi^{l_{a}}(z \mid \beta)$.

$$
|\lambda\rangle=\lim _{z \rightarrow 0} \phi^{l_{a}}(z \mid \beta)|0\rangle
$$

Here $|\lambda\rangle$ is the highest weight vector of the highest weight whose classical part $\bar{\lambda}=\sum_{i=1}^{N} l_{a}^{i} \bar{\Lambda}_{i}$.

The elementary vertex operators $\phi^{l_{a}}(z \mid \beta)$ give rise to the following map.

$$
\phi^{l_{a}}(z \mid \beta): F\left(p_{a}\right) \longrightarrow F\left(p_{a}+l_{a}\right) .
$$

Using the inversion relation,

$$
\sum_{r=1}^{N} \frac{\left[A_{i, r} m\right]}{[m]} \frac{[\operatorname{Min}(r, j) m][(N-1-\operatorname{Max}(r, j)) m]}{[(N-1) m][m]}=\delta_{i, j},
$$

we have the following proposition.

Proposition 5.7 The elementary vertex operators $\phi^{l_{a}}(z \mid \beta)$ satisfy the following relations.

$$
\begin{aligned}
& {\left[h_{i, m}, \phi^{l_{a}}(z \mid \beta)\right]=\frac{1}{m}\left[l_{a}^{i} m\right] q^{-\left(\beta+\frac{N-1}{2}\right)|m|} z^{m} \phi^{l_{a}}(z \mid \beta) \quad(1 \leq i \leq N),} \\
& {\left[X_{i}^{+}\left(z_{1}\right), \phi^{l_{a}}\left(z_{2} \mid \beta\right)\right]=0 \quad(1 \leq i \leq N),} \\
& \left(z_{1}-q^{l_{a}^{i}} z_{2}\right) X_{i}^{-}\left(z_{1}\right) \phi^{l_{a}}\left(z_{2} \mid-\frac{k+N-1}{2}\right) \\
& =\left(q^{l^{i}} z_{1}-z_{2}\right) \phi^{l_{a}}\left(z_{2} \mid-\frac{k+N-1}{2}\right) X_{i}^{-}\left(z_{1}\right) \quad(1 \leq i \leq N) .
\end{aligned}
$$

Proposition 5.8 For $k=\alpha \neq 0,-1,-2, \cdots,-N+1$, bosonizations of the components $\Phi_{2 N}(z)$ and $\Phi_{1}^{*}(z)$ associated with $U_{q}(\widehat{s l}(N \mid 1))$ are given by

$$
\Phi_{2^{N}}(z)=\phi^{\hat{l}}\left(q^{k+N-1} z \mid-\frac{k+N-1}{2}\right), \quad \Phi_{1}^{*}(z)=\phi^{\hat{l}^{*}}\left(q^{k} z \mid-\frac{k+N-1}{2}\right),
$$

where we have set $\hat{l}=-(0, \cdots, 0, \alpha+N-1)$ and $\hat{l}^{*}=(0, \cdots, 0, \alpha)$. The other components $\Phi_{j}(z)$ and $\Phi_{j}^{*}(z)\left(1 \leq j \leq 2^{N}\right)$ are represented by multiple contour integrals of Drinfeld currents (cf. propositions 5.2 and [5.3). We have checked this proposition for $N=2,3,4$.

These bosonizations of the vertex operators are determined from the commutation relations with the superalgebra $U_{q}(\widehat{s l}(N \mid 1))$. The construction is completely independent of which infinite dimensional 
modules the vertex operators intertwine. In what follow we shall clarify on which space these vertex operators act. We balance the "background charge" of the vertex operators by using the screening currents. For $x=\left(x_{1}, x_{2}, \cdots, x_{N}\right) \in \mathbf{N}^{N}$, we set the screening operator

$$
\mathcal{Q}^{(x)}=: Q_{1}^{x_{1}} Q_{2}^{x_{2}} \cdots Q_{N}^{x_{N}}:
$$

The screening operator $\mathcal{Q}^{(x)}$ give rise to the map,

$$
\mathcal{Q}^{(x)}: F\left(p_{a}\right) \longrightarrow F\left(p_{a}+\hat{x}\right)
$$

Here $\hat{x}=\left(\hat{x}_{1}, \hat{x}_{2}, \cdots, \hat{x}_{N}\right)$, where $\hat{x}_{i}=\sum_{j=1}^{N} A_{i, j} x_{j}$. The $\mathcal{Q}^{(x)}$ commute with the projection operator $\eta_{0} \xi_{0}$. Hence we have the map on the Fock-Wakimoto module.

$$
\mathcal{Q}^{(x)}: \mathcal{F}\left(p_{a}\right) \longrightarrow \mathcal{F}\left(p_{a}+\hat{x}\right)
$$

Definition 5.9 For $k=\alpha \neq 0,-1,-2, \cdots,-N$, we set the bosonic operators

$$
\widetilde{\Phi}^{(x)}(z)=\sum_{j=1}^{2^{N}} \widetilde{\Phi}_{j}^{(x)}(z) \otimes v_{j}, \quad \widetilde{\Phi}^{(y) *}(z)=\sum_{j=1}^{2^{N}} \widetilde{\Phi}_{j}^{(y) *}(z) \otimes v_{j}^{*} .
$$

Here we have set

$$
\begin{aligned}
\widetilde{\Phi}_{j}^{(x)}(z) & =\eta_{0} \xi_{0} \cdot \mathcal{Q}^{(x)} \cdot \Phi_{j}(z) \cdot \eta_{0} \xi_{0} \\
\widetilde{\Phi}_{j}^{(y) *}(z) & =\eta_{0} \xi_{0} \cdot \mathcal{Q}^{(y)} \cdot \Phi_{j}^{*}(z) \cdot \eta_{0} \xi_{0} .
\end{aligned}
$$

where $x=\left(x_{1}, x_{2}, \cdots, x_{N}\right) \in \mathbf{N}^{N}$ and $y=\left(y_{1}, y_{2}, \cdots, y_{N}\right) \in \mathbf{N}^{N}$. We call the operators $\widetilde{\Phi}^{(x)}(z), \widetilde{\Phi}^{(y) *}(z)$ the "projected vertex operators" for $U_{q}(\widehat{s l}(N \mid 1))$.

Proposition 5.10 For $k=\alpha \neq 0,-1,-2, \cdots,-N+1$, the projected vertex operators $\widetilde{\Phi}^{(x)}(z)$ and $\widetilde{\Phi}^{(y) *}(z)$ are the intertwiners among the Fock-Wakimoto module and the typical representation.

$$
\begin{aligned}
\widetilde{\Phi}^{(x)}(z) & : \quad \mathcal{F}\left(p_{a}\right) \longrightarrow \mathcal{F}\left(p_{a}+\hat{l}+\hat{x}\right) \otimes V_{\alpha, z}, \\
\widetilde{\Phi}^{(y) *}(z) & : \quad \mathcal{F}\left(p_{a}\right) \longrightarrow \mathcal{F}\left(p_{a}+\hat{l^{*}}+\hat{y}\right) \otimes V_{\alpha, z}^{* S} .
\end{aligned}
$$

Here we have set $\hat{l}=-(0, \cdots, 0, \alpha+N-1)$ and $\hat{l}^{*}=(0, \cdots, 0, \alpha)$. Here we have set $\hat{x}=\left(\hat{x}_{1}, \hat{x}_{2}, \cdots, \hat{x}_{N}\right)$ and $\hat{y}=\left(\hat{y}_{1}, \hat{y}_{2}, \cdots, \hat{y}_{N}\right)$ where $\hat{x}_{i}=\sum_{j=1}^{N} A_{i, j} x_{j}$ and $\hat{y}_{i}=\sum_{j=1}^{N} A_{i, j} y_{j}$. We have checked this proposition for $\operatorname{rank} N=2,3,4$.

\subsection{Correlation function}

In this section we discuss an application of the projected vertex operators $\widetilde{\Phi}^{(x)}(z)$ and $\widetilde{\Phi}^{*(y)}(z)$. We study non-vanishing property of the correlation function which is defined to be the trace of the vertex operators over the Fock-Wakimoto module of $U_{q}(\widehat{s l}(N \mid 1))$, that is

$$
\operatorname{Tr}_{\mathcal{F}\left(l_{a}\right)}\left(q^{L_{0}} \widetilde{\Phi}_{j_{1}}^{\left(x_{(1)}\right)}\left(z_{1}\right) \widetilde{\Phi}_{j_{2}}^{\left(x_{(2)}\right)}\left(z_{2}\right) \ldots \widetilde{\Phi}_{j_{n}}^{\left(x_{(n)}\right)}\left(z_{n}\right)\right) .
$$


Here we propose the $q$-Virasoro operator $L_{0}$ for $k=\alpha \neq-N+1$ as follows.

$$
\begin{aligned}
L_{0}= & \frac{1}{2} \sum_{i, j=1}^{N} \sum_{m \in \mathbf{Z}}: a_{-m}^{i} \frac{m^{2}}{[m][(k+N-1) m]} \frac{[\operatorname{Min}(i, j) m][(N-1-\operatorname{Max}(i, j)) m]}{[(N-1) m][m]} a_{m}^{j}: \\
& +\sum_{i, j=1}^{N} \frac{\operatorname{Min}(i, j)(N-1-\operatorname{Max}(i, j))}{(k+N-1)(N-1)} a_{0}^{j} \\
& -\frac{1}{2} \sum_{1 \leq i<j \leq N} \sum_{m \in \mathbf{Z}}: b_{-m}^{i, j} \frac{m^{2}}{[m]^{2}} b_{m}^{i, j}:+\frac{1}{2} \sum_{1 \leq i<j \leq N} \sum_{m \in \mathbf{Z}}: c_{-m}^{i, j} \frac{m^{2}}{[m]^{2}} c_{m}^{i, j}: \\
& +\frac{1}{2} \sum_{1 \leq i \leq N} \sum_{m \in \mathbf{Z}}: b_{-m}^{i, N+1} \frac{m^{2}}{[m]^{2}} b_{m}^{i, N+1}:+\frac{1}{2} \sum_{1 \leq i \leq N} b_{0}^{i, N+1} .
\end{aligned}
$$

The $L_{0}$ eigenvalue of $\left|l_{a}, 0,0\right\rangle$ is $\frac{1}{2(k+N-1)}(\bar{\lambda} \mid \bar{\lambda}+2 \bar{\rho})$, where $\bar{\rho}=\sum_{i=1}^{N} \bar{\Lambda}_{i}$ and $\bar{\lambda}=\sum_{i=1}^{N} l_{a}^{i} \bar{\Lambda}_{i}$.

Proposition 5.11 The correlation function of the vertex operators,

$$
\operatorname{Tr}_{\mathcal{F}\left(l_{a}\right)}\left(q^{L_{0}} \widetilde{\Phi}_{j_{1}}^{\left(x_{(1)}\right)}\left(z_{1}\right) \widetilde{\Phi}_{j_{2}}^{\left(x_{(2)}\right)}\left(z_{2}\right) \cdots \widetilde{\Phi}_{j_{n}}^{\left(x_{(n)}\right)}\left(z_{n}\right)\right) \neq 0
$$

if and only if $k=\alpha \neq 0,-1,-2, \cdots,-N+1$ and $x_{(s)}=\left(x_{(s), 1}, x_{(s), 2} \cdots, x_{(s), N}\right) \in \mathbf{N}^{N}(1 \leq s \leq n)$ satisfy the condition,

$$
\sum_{s=1}^{n} x_{(s), i}=\frac{n \cdot i}{N-1} \alpha+n \cdot i \quad(1 \leq i \leq N) .
$$

We note that there doesn't exist non-rational solution $k=\alpha \notin \mathbf{Q}$ of the relation (5.108). Next, we consider the correlation function involving also dual vertex operators.

Proposition 5.12 The correlation function of the vertex operators and the dual vertex operators,

$$
\operatorname{Tr}_{\mathcal{F}\left(l_{a}\right)}\left(q^{L_{0}} \widetilde{\Phi}_{i_{1}}^{*\left(y_{(1)}\right)}\left(w_{1}\right) \widetilde{\Phi}_{i_{2}}^{*\left(y_{(2)}\right)}\left(w_{2}\right) \ldots \widetilde{\Phi}_{i_{m}}^{*\left(y_{(m)}\right)}\left(w_{m}\right) \widetilde{\Phi}_{j_{1}}^{\left(x_{(1)}\right)}\left(z_{1}\right) \widetilde{\Phi}_{j_{2}}^{\left(x_{(2)}\right)}\left(z_{2}\right) \ldots \widetilde{\Phi}_{j_{n}}^{\left(x_{(n)}\right)}\left(z_{n}\right)\right) \neq 0
$$

if and only if $k=\alpha \neq 0,-1,-2, \cdots,-N+1, x_{(s)}=\left(x_{(s), 1}, x_{(s), 2}, \cdots, x_{(s), N}\right) \in \mathbf{N}^{N}(1 \leq s \leq n)$ and $y_{(t)}=\left(y_{(t), 1}, y_{(t), 2}, \cdots, y_{(t), N}\right) \in \mathbf{N}^{N}(1 \leq t \leq m)$ satisfy the condition

$$
\sum_{s=1}^{n} x_{(s), i}+\sum_{t=1}^{m} y_{(t), i}=\frac{(n-m) i}{N-1} \alpha+n \cdot i \quad(1 \leq i \leq N) .
$$

We note that there exist non-rational solutions $k=\alpha \notin \mathbf{Q}$ of the relation (5.110). Upon $k=\alpha \notin \mathbf{Q}$, the relation (5.110) is equivalent to

$$
m=n \quad \text { and } \quad \sum_{s=1}^{n}\left(x_{(s), i}+y_{(s), i}\right)=n \cdot i \quad(1 \leq i \leq N) .
$$

We conclude that the screening operators $Q_{i}$ are needed to ensure non-vanishing property of correlation functions. In other words, we have to balance the "background charge" of the vertex operators to construct non-zero correlation functions. We can write down integral representations of the correlation functions by using bosonizations of the vertex operators [22]. It is open and nontrivial problem to deform these integral representations to convenient formulae for physical applications. 


\section{Acknowledgements}

This work is supported by the Grant-in-Aid for Scientific Research C (21540228) from Japan Society for Promotion of Science. The author would like to thank Professor Etsuro Date, Professor Hiroyuki Yamane, Professor Masato Okado, Professor Kenji Iohara, and Professor Hitoshi Konno for their interests to this work.

\section{A Normal orderings}

In this appendix we summarize formulae of normal orderings. In order to get the following delta-function formulae, the following normal orderings are useful.

$$
\begin{aligned}
& \exp \left(a_{+}^{i}\left(q^{\frac{k+N-1}{2}} z_{1}\right)\right): \exp \left(-\left(\frac{1}{k+N-1} a^{j}\right)\left(z_{2} \mid \frac{k+N-1}{2}\right)\right): \\
& =:: q^{-A_{i, j}} \frac{\left(1-q^{A_{i, j}-k-N+1} z_{2} / z_{1}\right)}{\left(1-q^{-A_{i, j}-k-N+1} z_{2} / z_{1}\right)}, \\
& : \exp \left(-\left(\frac{1}{k+N-1} a^{j}\right)\left(z_{2} \mid \frac{k+N-1}{2}\right)\right): \exp \left(a_{+}^{i}\left(q^{\frac{k+N-1}{2}} z_{1}\right)\right)=:: 1, \\
& : \exp \left(-\left(\frac{1}{k+N-1} a^{i}\right)\left(z_{1} \mid \frac{k+N-1}{2}\right)\right): \exp \left(a_{-}^{j}\left(q^{-\frac{k+N-1}{2}} z_{2}\right)\right) \\
& =:: \frac{\left(1-q^{A_{i, j}-k-N+1} z_{2} / z_{1}\right)}{\left(1-q^{-A_{i, j}-k-N+1} z_{2} / z_{1}\right)}, \\
& \exp \left(a_{-}^{i}\left(q^{-\frac{k+N-1}{2}} z_{2}\right)\right): \exp \left(-\left(\frac{1}{k+N-1} a^{j}\right)\left(z_{1} \mid \frac{k+N-1}{2}\right)\right):=:: q^{A_{i, j}} .
\end{aligned}
$$

In order to get the following specialization relations, the following formula is useful.

$$
b^{i, j}(q z)-b^{i, j}\left(q^{-1} z\right)=b_{+}^{i, j}(z)-b_{-}^{i, j}(z)
$$

\section{A.1 Delta-function}

$$
\begin{aligned}
& \quad\left[X_{i}^{-(N, 0)}\left(z_{1}\right), S_{i}^{(N+1,0)}\left(z_{2}\right)\right] \\
& =\frac{-1}{\left(q-q^{-1}\right) q^{k+N} z_{1} z_{2}}\left(\delta\left(\frac{q^{-N-k+1} z_{2}}{z_{1}}\right)-\delta\left(\frac{q^{-N-k-1} z_{2}}{z_{1}}\right)\right):: \quad(1 \leq i \leq N-1), \\
& \quad\left[X_{i}^{-(i, 1)}\left(z_{1}\right), S_{i}^{(i+1,1)}\left(z_{2}\right)\right]=\left(q-q^{-1}\right) \delta\left(\frac{q^{N+k-1} z_{2}}{z_{1}}\right):: \quad(1 \leq i \leq N-1), \\
& {\left[X_{i}^{-(N-1,1)}\left(z_{1}\right), S_{i}^{(N, 2)}\left(z_{2}\right)\right]=\left(q^{-1}-q\right) \delta\left(\frac{q^{-N-k-1} z_{2}}{z_{1}}\right):: \quad(1 \leq i \leq N-1),} \\
& {\left[X_{i}^{-(l+1,2)}\left(z_{1}\right), S_{i}^{(l+2,1)}\left(z_{2}\right)\right]=\left(q-q^{-1}\right) \delta\left(\frac{q^{N-3-k-2 l} z_{2}}{z_{1}}\right):: \quad(1 \leq i \leq l \leq N-2),}
\end{aligned}
$$




$$
\begin{aligned}
& {\left[X_{i}^{-(l+1,1)}\left(z_{1}\right), S_{i}^{(l+2,2)}\left(z_{2}\right)\right]=\left(q^{-1}-q\right) \delta\left(\frac{q^{N-3-k-2 l} z_{2}}{z_{1}}\right):: \quad(1 \leq i \leq l \leq N-3),} \\
& {\left[X_{i}^{-(i, 2)}\left(z_{1}\right), S_{i}^{(i+1,2)}\left(z_{2}\right)\right]=\left(q^{-1}-q\right) \delta\left(\frac{q^{N-3-k-2 i} z_{2}}{z_{1}}\right):: \quad(1 \leq i \leq N-2)} \\
& {\left[X_{N}^{-(N, 1)}\left(z_{1}\right), S_{N}^{(N+1,0)}\left(z_{2}\right)\right]=\frac{1}{q^{-N-k+1} z_{1}} \delta\left(\frac{q^{N+k-1} z_{2}}{z_{1}}\right)::} \\
& {\left[X_{N}^{-(N, 2)}\left(z_{1}\right), S_{N}^{(N+1,0)}\left(z_{2}\right)\right]=\frac{1}{q^{N+k-1} z_{1}} \delta\left(\frac{q^{-N-k+1} z_{2}}{z_{1}}\right)::} \\
& {\left[X_{i}^{-(l, 1)}\left(z_{1}\right), S_{i+1}^{(l+1,2)}\left(z_{2}\right)\right]=\left(q-q^{-1}\right) \delta\left(\frac{q^{N-k-2 l-2} z_{2}}{z_{1}}\right):: \quad(1 \leq i \leq N-2, i+1 \leq l \leq N-1),} \\
& {\left[X_{i}^{-(l, 2)}\left(z_{1}\right), S_{i+1}^{(l+1,1)}\left(z_{2}\right)\right]=\left(q^{-1}-q\right) \delta\left(\frac{q^{N-k-2 l-2} z_{2}}{z_{1}}\right):: \quad(1 \leq i \leq N-2, i+1 \leq l \leq N-1),} \\
& {\left[X_{i}^{-(j, 1)}\left(z_{1}\right), S_{j}^{(i+1,1)}\left(z_{2}\right)\right]=\left(q-q^{-1}\right) \delta\left(\frac{q^{N+k-i+j-1} z_{2}}{z_{1}}\right):: \quad(1 \leq j<i \leq N-1),} \\
& {\left[X_{i}^{-(j+1,1)}\left(z_{1}\right), S_{j}^{(i, 1)}\left(z_{2}\right)\right]=\left(q^{-1}-q\right) \delta\left(\frac{q^{N+k-i+j-1} z_{2}}{z_{1}}\right):: \quad(1 \leq j<i \leq N)} \\
& {\left[X_{i}^{-(j, 2)}\left(z_{1}\right), S_{j}^{(i+1,1)}\left(z_{2}\right)\right]=\left(q-q^{-1}\right) \delta\left(\frac{q^{N+k-i+j-1} z_{2}}{z_{1}}\right):: \quad(1 \leq j<i \leq N-1),} \\
& {\left[X_{i}^{-(j+1,1)}\left(z_{1}\right), S_{j}^{(i, 2)}\left(z_{2}\right)\right]=\left(q^{-1}-q\right) \delta\left(\frac{q^{N+k-i+j-1} z_{2}}{z_{1}}\right):: \quad(1 \leq j<i \leq N),} \\
& {\left[X_{i}^{-(j, 1)}\left(z_{1}\right), S_{j}^{(i, 2)}\left(z_{2}\right)\right]=\left(1-q^{-2}\right) \delta\left(\frac{q^{N+k-i+j-1} z_{2}}{z_{1}}\right):: \quad(1 \leq j<i \leq N),} \\
& {\left[X_{i}^{-(j, 2)}\left(z_{1}\right), S_{j}^{(i, 1)}\left(z_{2}\right)\right]=\left(q^{-2}-1\right) \delta\left(\frac{q^{N+k-i+j-1} z_{2}}{z_{1}}\right):: \quad(1 \leq j<i \leq N),} \\
& {\left[X_{N}^{-(j, 1)}\left(z_{1}\right), S_{j}^{(N+1,0)}\left(z_{2}\right)\right]=\frac{1}{q^{-k-j+1} z_{1}} \delta\left(\frac{q^{k+j-1} z_{2}}{z_{1}}\right):: \quad(1 \leq j \leq N-1),} \\
& {\left[X_{N}^{-(j, 2)}\left(z_{1}\right), S_{j}^{(N+1,0)}\left(z_{2}\right)\right]=\frac{1}{q^{-k-j+1} z_{1}} \delta\left(\frac{q^{k+j-1} z_{2}}{z_{1}}\right):: \quad(1 \leq j \leq N-1),} \\
& {\left[X_{i}^{+(i, 1)}\left(z_{1}\right), S_{i}^{(i+1,2)}\left(z_{2}\right)\right]=\left(q-q^{-1}\right) \delta\left(\frac{q^{N-2 i-1} z_{2}}{z_{1}}\right):: \quad(1 \leq i \leq N-1),}
\end{aligned}
$$




$$
\begin{gathered}
{\left[X_{i}^{+(i, 2)}\left(z_{1}\right), S_{i}^{(i+1,1)}\left(z_{2}\right)\right]=\left(q^{-1}-q\right) \delta\left(\frac{q^{N-2 i-1} z_{2}}{z_{1}}\right):: \quad(1 \leq i \leq N-1)} \\
{\left[X_{i}^{+(j, 1)}\left(z_{1}\right), S_{j}^{(i, 2)}\left(z_{2}\right)\right]=\left(1-q^{2}\right) \delta\left(\frac{q^{N-i-j-1} z_{2}}{z_{1}}\right):: \quad(1 \leq i \leq N, 1 \leq j \leq i-1),} \\
{\left[X_{i}^{+(j+1,2)}\left(z_{1}\right), S_{j}^{(i+1,1)}\left(z_{2}\right)\right]=\left(q^{2}-1\right) \delta\left(\frac{q^{N-i-j-1} z_{2}}{z_{1}}\right):: \quad(1 \leq i \leq N, 1 \leq j \leq i-1),} \\
{\left[X_{N}^{+(j, 0)}\left(z_{1}\right), S_{j}^{(N, 2)}\left(z_{2}\right)\right]=\left(1-q^{2}\right) \delta\left(\frac{q^{-j-1} z_{2}}{z_{1}}\right):: \quad(1 \leq j \leq N-1),} \\
{\left[X_{i}^{+(j, 1)}\left(z_{1}\right), S_{j}^{(i+1,2)}\left(z_{2}\right)\right]=\left(q-q^{-1}\right) \delta\left(\frac{q^{N-i-j-1} z_{2}}{z_{1}}\right):: \quad(1 \leq i \leq N-1,1 \leq j \leq i-1),(1 \leq j \leq N-1),} \\
{\left[X_{i}^{+(j+1,2)}\left(z_{1}\right), S_{j}^{(i+1,2)}\left(z_{2}\right)\right]=\left(q^{2}-1\right) \delta\left(\frac{q^{N-i-j-1} z_{2}}{z_{1}}\right):: \quad(1 \leq i \leq N-1,1 \leq j \leq i-1),} \\
{\left[X_{i}^{+(j, 2)}\left(z_{1}\right), S_{j}^{(i+1,1)}\left(z_{2}\right)\right]=\left(q^{-1}-q\right) \delta\left(\frac{q^{N-i-j-1} z_{2}}{z_{1}}\right):: \quad(1 \leq i \leq N-1,1 \leq j \leq i-1),} \\
{\left[X_{i}^{+(j, 2)}\left(z_{1}\right), S_{j}^{(i, 2)}\left(z_{2}\right)\right]=\left(1-q^{2}\right) \delta\left(\frac{q^{N-i-j-1} z_{2}}{z_{1}}\right):: \quad(1 \leq i \leq N-1,1 \leq j \leq i-1),}
\end{gathered}
$$

\section{A.2 Specialization}

$$
\begin{gathered}
: X_{i}^{-(N, 0)}(z) S_{i}^{(N+1,0)}\left(q^{N+k-1} z\right):=: X_{i}^{-(i, 1)}(z) S_{i}^{(i+1,1)}\left(q^{-N-k+1} z\right): \\
=: \exp \left(-\left(\frac{1}{k+N-1} a^{i}\right)\left(z \mid-\frac{k+N-1}{2}\right)\right): \quad(1 \leq i \leq N-1), \\
: X_{N}^{-(N, 1)}(z) S_{N}^{(N+1,0)}\left(q^{-N-k+1} z\right):=: X_{N}^{-(N, 2)}(z) S_{N}^{(N+1,0)}\left(q^{N+k-1} z\right): \\
=: \exp \left(-\left(\frac{1}{k+N-1} a^{i}\right)\left(z \mid-\frac{k+N-1}{2}\right)\right): \\
: X_{i}^{-(N, 0)}(z) S_{i}^{(N+1,0)}\left(q^{N+k+1} z\right):=: X_{i}^{-(N-1,1)}(z) S_{i}^{(N, 2)}\left(q^{N+k+1} z\right): \\
\quad(1 \leq i \leq N-1), \\
: X_{i}^{-(i+1,2)}(z) S_{i}^{(i+2,1)}\left(q^{-N+3+k+2 i} z\right):=: X_{i}^{-(i, 2)}(z) S_{i}^{(i+1,2)}\left(q^{-N+3+k+2 i} z\right): \\
\quad(1 \leq i \leq N-1),
\end{gathered}
$$




$$
\begin{aligned}
& : X_{i}^{-(l+1,1)}(z) S_{i}^{(l+2,2)}\left(q^{-N+3+k+2 l} z\right):=: X_{i}^{-(l+1,2)}(z) S_{i}^{(l+2,1)}\left(q^{-N+3+k+2 l} z\right): \\
& (1 \leq i \leq N-1, i+1 \leq l \leq N-2) . \\
& : X_{i}^{-(l, 1)}(z) S_{i+1}^{(l+1,2)}\left(q^{-N+k+2 l+2} z\right):=: X_{i}^{-(l, 2)}(z) S_{i+1}^{(l+1,1)}\left(q^{-N+k+2 l+2} z\right):, \\
& (1 \leq i \leq N-2, i+1 \leq l \leq N-1) \\
& : X_{i}^{-(j, 1)}(z) S_{j}^{(i+1,1)}\left(q^{-N-k+i-j+1} z\right):=: X_{i}^{-(j+1,1)}(z) S_{j}^{(i, 1)}\left(q^{-N-k+i-j+1} z\right): \\
& (1 \leq j<i \leq N-1) \\
& : X_{i}^{-(j, 2)}(z) S_{j}^{(i+1,1)}\left(q^{-N-k+i-j+1} z\right):=: X_{i}^{-(j+1,1)}(z) S_{j}^{(i, 2)}\left(q^{-N-k+i-j+1} z\right): \\
& (1 \leq j<i \leq N-1), \\
& : X_{i}^{-(j, 2)}(z) S_{j}^{(i, 1)}\left(q^{-N-k+i-j+1} z\right):=: X_{i}^{-(j, 1)}(z) S_{j}^{(i, 2)}\left(q^{-N-k+i-j+1} z\right): \\
& (1 \leq j<i \leq N) \\
& : X_{N}^{-(j, 1)}(z) S_{j}^{(N+1,0)}\left(q^{-k-j+1} z\right):=: X_{N}^{-(j+1,1)}(z) S_{j}^{(N, 1)}\left(q^{-k-j+1} z\right): \\
& (1 \leq j<i \leq N-1) \text {, } \\
& : X_{N}^{-(j, 2)}(z) S_{j}^{(N+1,0)}\left(q^{-k-j+1} z\right):=: X_{N}^{-(j+1,1)}(z) S_{j}^{(N, 2)}\left(q^{-k-j+1} z\right): \\
& (1 \leq j<i \leq N-1) \\
& : X_{i}^{+(i, 1)}(z) S_{i}^{(i+1,2)}\left(q^{-N+2 i+1} z\right):=: X_{i}^{+(i, 2)}(z) S_{i}^{(i+1,1)}\left(q^{-N+2 i+1} z\right): \\
& (1 \leq i \leq N-1) \text {, } \\
& : X_{i}^{+(j, 1)}(z) S_{j}^{(i, 2)}\left(q^{-N+i+j+1} z\right):=: X_{i}^{+(j+1,2)}(z) S_{j}^{(i+1,1)}\left(q^{-N+i+j+1} z\right): \\
& (1 \leq i \leq N-1,1 \leq j \leq i-1), \\
& : X_{N}^{+(j, 0)}(z) S_{j}^{(N, 2)}\left(q^{j+1} z\right):=: X_{N}^{+(j+1,0)}(z) S_{j}^{(N+1,0)}\left(q^{j+1} z\right): \\
& (1 \leq j \leq N-1) \\
& : X_{i}^{+(j, 1)}(z) S_{j}^{(i+1,2)}\left(q^{-N+i+j+1} z\right):=: X_{i}^{+(j, 2)}(z) S_{j}^{(i+1,1)}\left(q^{-N+i+j+1} z\right): \\
& (1 \leq i \leq N-1,1 \leq j \leq i-1), \\
& : X_{i}^{+(j, 2)}(z) S_{j}^{(i, 2)}\left(q^{-N+i+j+1} z\right):=: X_{i}^{+(j+1,2)}(z) S_{j}^{(i+1,2)}\left(q^{-N+i+j+1} z\right): \\
& (1 \leq i \leq N-1,1 \leq j \leq i-1),
\end{aligned}
$$




\section{B Level-zero representation of $U_{q}(\widehat{s l}(4 \mid 1))$}

In this appendix we summarize the level-zero representation of $U_{q}(\widehat{s l}(4 \mid 1))$. Let $V_{\alpha}$ be the one parameter family of the $16\left(=2^{4}\right)$-dimensional typical representation of $U_{q}(\operatorname{sl}(4 \mid 1))$ [36, 37. In the case of $U_{q}(\operatorname{sl}(4 \mid 1))$, we choose the basis $\left\{v_{j}\right\}_{1 \leq j \leq 16}$ of $V_{\alpha}$ and assign them the $\mathbf{Z}_{2}$-gradings as following.

$$
\begin{aligned}
& \left|v_{1}\right|=\left|v_{5}\right|=\left|v_{7}\right|=\left|v_{8}\right|=\left|v_{9}\right|=\left|v_{10}\right|=\left|v_{12}\right|=\left|v_{16}\right|=0, \\
& \left|v_{2}\right|=\left|v_{3}\right|=\left|v_{4}\right|=\left|v_{6}\right|=\left|v_{11}\right|=\left|v_{13}\right|=\left|v_{14}\right|=\left|v_{15}\right|=1 .
\end{aligned}
$$

In the homogeneous gradation, the evaluation representation $V_{\alpha, z}$ of $U_{q}(\widehat{s l}(4 \mid 1))$ is given by

$$
\begin{aligned}
& h_{1}=E_{4,4}-E_{6,6}+E_{7,7}-E_{8,8}+E_{9,9}-E_{10,10}+E_{11,11}-E_{13,13}, \\
& h_{2}=E_{3,3}-E_{4,4}+E_{5,5}-E_{7,7}+E_{10,10}-E_{12,12}+E_{13,13}-E_{14,14} \text {, } \\
& h_{3}=E_{2,2}-E_{3,3}+E_{7,7}-E_{9,9}+E_{8,8}-E_{10,10}+E_{14,14}-E_{15,15}, \\
& h_{4}=\alpha \sum_{j=1}^{2} E_{j, j}+(\alpha+1) \sum_{j=3}^{8} E_{j, j}+(\alpha+2) \sum_{j=9}^{14} E_{j, j}+(\alpha+3) \sum_{j=15}^{16} E_{j, j} \text {, } \\
& e_{1}=E_{4,6}+E_{7,8}+E_{9,10}+E_{11,13} \text {, } \\
& e_{2}=E_{3,4}+E_{5,7}+E_{10,12}+E_{13,14} \\
& e_{3}=E_{2,3}+E_{7,9}+E_{8,10}+E_{14,15} \text {, } \\
& e_{4}=-\sqrt{[\alpha]} E_{1,2}+\sqrt{[\alpha+1]}\left(E_{3,5}+E_{4,7}+E_{6,8}\right) \\
& -\sqrt{[\alpha+2]}\left(E_{9,11}+E_{10,13}+E_{12,14}\right)+\sqrt{[\alpha+3]} E_{15,16}, \\
& f_{1}=E_{6,4}+E_{8,7}+E_{10,9}+E_{13,11}, \\
& f_{2}=E_{4,3}+E_{7,5}+E_{12,10}+E_{14,13}, \\
& f_{3}=E_{3,2}+E_{9,7}+E_{10,8}+E_{15,14}, \\
& f_{4}=-\sqrt{[\alpha]} E_{2,1}+\sqrt{[\alpha+1]}\left(E_{5,3}+E_{7,4}+E_{8,6}\right) \\
& -\sqrt{[\alpha+2]}\left(E_{11,9}+E_{13,10}+E_{14,12}\right)+\sqrt{[\alpha+3]} E_{16,15}, \\
& h_{0}=-\alpha\left(E_{1,1}+E_{6,6}\right)-(\alpha+1)\left(E_{2,2}+E_{3,3}+E_{4,4}+E_{8,8}+E_{10,10}+E_{12,12}\right) \\
& -(\alpha+2)\left(E_{5,5}+E_{7,7}+E_{9,9}+E_{13,13}+E_{14,14}+E_{15,15}\right)-(\alpha+3)\left(E_{11,11}+E_{16,16}\right), \\
& e_{0}=z\left(\sqrt{[\alpha]} E_{6,1}-\sqrt{[\alpha+1]}\left(E_{8,2}+E_{10,3}+E_{12,4}\right)\right. \\
& \left.+\sqrt{[\alpha+2]}\left(E_{13,5}+E_{14,7}+E_{15,9}\right)-\sqrt{[\alpha+3]} E_{16,11}\right), \\
& f_{0}=-z^{-1}\left(\sqrt{[\alpha]} E_{1,6}-\sqrt{[\alpha+1]}\left(E_{2,8}+E_{3,10}+E_{4,12}\right)\right. \\
& \left.+\sqrt{[\alpha+2]}\left(E_{5,13}+E_{7,14}+E_{9,15}\right)-\sqrt{[\alpha+3]} E_{11,16}\right) \text {. }
\end{aligned}
$$

We choose the dual basis $\left\{v_{j}^{*}\right\}_{1 \leq j \leq 16}$ of $V_{\alpha}^{*, S}$ and assign them the $\mathbf{Z}_{2}$-gradings as following.

$$
\begin{aligned}
& \left|v_{1}^{*}\right|=\left|v_{5}^{*}\right|=\left|v_{7}^{*}\right|=\left|v_{8}^{*}\right|=\left|v_{9}^{*}\right|=\left|v_{10}^{*}\right|=\left|v_{12}^{*}\right|=\left|v_{16}^{*}\right|=0, \\
& \left|v_{2}^{*}\right|=\left|v_{3}^{*}\right|=\left|v_{4}^{*}\right|=\left|v_{6}^{*}\right|=\left|v_{11}^{*}\right|=\left|v_{13}^{*}\right|=\left|v_{14}^{*}\right|=\left|v_{15}^{*}\right|=1 .
\end{aligned}
$$


In the homogeneous gradation, the evaluation representation $V_{\alpha, z}^{* S}$ of $U_{q}(\widehat{s l}(4 \mid 1))$ is given by

$$
\begin{aligned}
h_{1}= & -E_{4,4}+E_{6,6}-E_{7,7}+E_{8,8}-E_{9,9}+E_{10,10}-E_{11,11}+E_{13,13} \\
h_{2}= & -E_{3,3}+E_{4,4}-E_{5,5}+E_{7,7}-E_{10,10}+E_{12,12}-E_{13,13}+E_{14,14} \\
h_{3}= & -E_{2,2}+E_{3,3}-E_{7,7}+E_{9,9}-E_{8,8}+E_{10,10}-E_{14,14}+E_{15,15} \\
h_{4}= & -\alpha \sum_{j=1}^{2} E_{j, j}-(\alpha+1) \sum_{j, j}-(\alpha+2) \sum_{j=9}^{14} E_{j, j}-(\alpha+3) \sum_{j=15} E_{j, j} \\
e_{1}= & -q^{-1}\left(E_{6,4}+E_{8,7}+E_{10,9}+E_{13,11}\right) \\
e_{2}= & -q^{-1}\left(E_{4,3}+E_{7,5}+E_{12,10}+E_{14,13}\right) \\
e_{3}= & -q^{-1}\left(E_{3,2}+E_{9,7}+E_{10,8}+E_{15,14}\right) \\
e_{4}= & \sqrt{[\alpha]} q^{-\alpha} E_{2,1}+\sqrt{[\alpha+1]} q^{-\alpha-1}\left(E_{5,3}+E_{7,4}+E_{8,6}\right) \\
& +\sqrt{[\alpha+2]} q^{-\alpha-2}\left(E_{11,9}+E_{13,10}+E_{14,12}\right)+\sqrt{[\alpha+3]} q^{-\alpha-3} E_{16,15} \\
f_{1}= & -q\left(E_{4,6}+E_{7,8}+E_{9,10}+E_{11,13}\right) \\
f_{2}= & -q\left(E_{3,4}+E_{5,7}+E_{10,12}+E_{13,14}\right) \\
f_{3}= & -q\left(E_{2,3}+E_{7,9}+E_{8,10}+E_{14,15}\right) \\
f_{4}= & -\sqrt{[\alpha]} q^{\alpha} E_{1,2}-\sqrt{[\alpha+1]} q^{\alpha+1}\left(E_{3,5}+E_{4,7}+E_{6,8}\right) \\
& -\sqrt{[\alpha+2]} q^{\alpha+2}\left(E_{9,11}+E_{10,13}+E_{12,14}\right)-\sqrt{[\alpha+3]} q^{\alpha+3} E_{15,16} \\
& \left.+\sqrt{[\alpha+2]} q^{-\alpha-2}\left(E_{13,5}+E_{14,7}+E_{15,9}\right)+\sqrt{[\alpha+3]} q^{-\alpha-3} E_{16,11}\right) \\
& +\left(\alpha+E_{1,1}+E_{6,6}\right)+(\alpha+1)\left(E_{2,2}+E_{3,3}+E_{4,4}+E_{8,8}+E_{10,10}+E_{12,12}\right) \\
h_{0}=\left(E_{5,5}+E_{7,7}+E_{9,9}+E_{13,13}+E_{14,14}+E_{15,15}\right)+(\alpha+3)\left(E_{11,11}+E_{16,16}\right) & z^{-1}\left(\sqrt{[\alpha]} q^{-\alpha} E_{6,1}+\sqrt{[\alpha+1]} q^{-\alpha-1}\left(E_{8,2}+E_{10,3}+E_{12,4}\right)\right. \\
f_{0}= & z\left(\sqrt{[\alpha]} q^{\alpha} E_{1,6}+\sqrt{[\alpha+1]} q^{\alpha+1}\left(E_{2,8}+E_{3,10}+E_{4,12}\right)\right. \\
& \left.+\sqrt{[\alpha+2}\left(E_{5,13}+E_{7,14}+E_{9,15}\right)+\sqrt{[\alpha+3]} q^{\alpha+3} E_{11,16}\right) \\
= & \\
= & \\
= &
\end{aligned}
$$

We give the level-zero realization of the Drinfeld generators.

Proposition B.1 On $V_{\alpha, z}$, the Drinfeld generators of $U_{q}(\widehat{s l}(4 \mid 1))$ are given by

$$
\begin{aligned}
h_{1, m} & =\frac{[m]}{m}\left(q^{\alpha+3} z\right)^{m}\left(q^{-m} E_{4,4}-q^{m} E_{6,6}+q^{-m} E_{7,7}-q^{m} E_{8,8}\right. \\
& \left.+q^{-m} E_{9,9}-q^{m} E_{10,10}+q^{-m} E_{11,11}-q^{m} E_{13,13}\right), \\
h_{2, m} & =\frac{[m]}{m}\left(q^{\alpha+3} z\right)^{m}\left(q^{-2 m} E_{3,3}-E_{4,4}+q^{-2 m} E_{5,5}-E_{7,7}\right. \\
& \left.+E_{10,10}-q^{2 m} E_{12,12}+E_{13,13}-q^{2 m} E_{14,14}\right), \\
h_{3, m} & =\frac{[m]}{m}\left(q^{\alpha+3} z\right)^{m}\left(q^{-3 m} E_{2,2}-q^{-m} E_{3,3}+q^{-m} E_{7,7}-q^{m} E_{9,9}\right. \\
& \left.+q^{-m} E_{8,8}-q^{m} E_{10,10}+q^{m} E_{14,14}-q^{3 m} E_{15,15}\right), \\
h_{4, m} & =\frac{1}{m} z^{m}\left([\alpha m] \sum_{j=1}^{2} E_{j, j}+[(\alpha+1) m] q^{m} \sum_{j=3}^{8} E_{j, j}\right.
\end{aligned}
$$




$$
\begin{aligned}
& \left.+[(\alpha+2) m] q^{2 m} \sum_{j=9}^{14} E_{j, j}+[(\alpha+3) m] q^{3 m} \sum_{j=15}^{16} E_{j, j}\right), \\
x_{1, n}^{+} & =\left(q^{\alpha+3} z\right)^{m}\left(E_{4,6}+E_{7,8}+E_{9,10}+E_{11,13}\right), \\
x_{2, n}^{+} & =\left(q^{\alpha+3} z\right)^{n}\left(q^{-3 n} E_{3,4}+q^{-n} E_{5,7}+q^{n} E_{10,12}+q^{n} E_{13,14}\right), \\
x_{3, n}^{+} & =\left(q^{\alpha+3} z\right)^{n}\left(q^{-2 n} E_{2,3}+E_{7,9}+E_{8,10}+q^{2 n} E_{14,15}\right), \\
x_{4, n}^{+} & =\left(q^{\alpha+3} z\right)^{n}\left(-\sqrt{[\alpha]} q^{-3 n} E_{1,2}+\sqrt{[\alpha+1]} q^{-n}\left(E_{3,5}+E_{4,7}+E_{6,8}\right)\right. \\
& \left.-\sqrt{[\alpha+2]_{q}} q^{n}\left(E_{9,11}+E_{10,13}+E_{12,14}\right)+\sqrt{[\alpha+3]} q^{3 n} E_{15,16}\right), \\
x_{1, n}^{-} & =\left(q^{\alpha+3} z\right)^{m}\left(E_{6,4}+E_{8,7}+E_{10,9}+E_{13,11}\right), \\
x_{2, n}^{-} & =\left(q^{\alpha+3} z\right)^{n}\left(q^{-n} E_{4,3}+q^{-n} E_{7,5}+q^{n} E_{12,10}+q^{n} E_{14,13}\right), \\
x_{3, n}^{-} & =\left(q^{\alpha+3} z\right)^{n}\left(q^{-2 n} E_{3,2}+E_{9,7}+E_{10,8}+q^{2 n} E_{15,14}\right), \\
x_{4, n}^{-} & =\left(q^{\alpha+3} z\right)^{n}\left(-\sqrt{[\alpha]} q^{-3 n} E_{2,1}+\sqrt{[\alpha+1]} q^{-n}\left(E_{5,3}+E_{7,4}+E_{8,6}\right)\right. \\
& \left.-\sqrt{[\alpha+2]} q^{n}\left(E_{11,9}+E_{13,10}+E_{14,12}\right)+\sqrt{[\alpha+3]} q^{3 n} E_{16,15}\right) .
\end{aligned}
$$

On $V_{\alpha, z}^{* S}$, the Drinfeld generators of $U_{q}(\widehat{s l}(4 \mid 1))$ are given by

$$
\begin{aligned}
h_{1, m}= & \frac{[m]}{m}\left(q^{-\alpha-3} z\right)^{m}\left(-q^{m} E_{4,4}+q^{-m} E_{6,6}-q^{m} E_{7,7}+q^{-m} E_{8,8}\right. \\
& \left.-q^{m} E_{9,9}+q^{-m} E_{10,10}-q^{m} E_{11,11}+q^{-m} E_{13,13}\right), \\
h_{2, m}= & \frac{[m]}{m}\left(q^{-\alpha-3} z\right)^{m}\left(-q^{2 m} E_{3,3}+E_{4,4}-q^{2 m} E_{5,5}+E_{7,7}\right. \\
& \left.-E_{10,10}+q^{-2 m} E_{12,12}-E_{13,13}+q^{-2 m} E_{14,14}\right), \\
h_{3, m}= & \frac{[m]}{m}\left(q^{-\alpha-3} z\right)^{m}\left(-q^{3 m} E_{2,2}+q^{m} E_{3,3}-q^{m} E_{7,7}+q^{-m} E_{9,9}\right. \\
& \left.-q^{m} E_{8,8}+q^{-m} E_{10,10}-q^{-m} E_{14,14}+q^{-3 m} E_{15,15}\right), \\
h_{4, m}= & -1 \\
& m z^{m}\left([\alpha m] \sum_{j=1}^{2} E_{j, j}+[(\alpha+1) m] q^{-m} \sum_{j=3}^{8} E_{j, j}\right. \\
& \left.+[(\alpha+2) m] q^{-2 m} \sum_{j=9}^{14} E_{j, j}+[(\alpha+3) m] q^{-3 m} \sum_{j=15}^{16} E_{j, j}\right), \\
x_{1, n}^{+}= & -q^{-1}\left(q^{-\alpha-3} z\right)^{n}\left(E_{6,4}+E_{8,7}+E_{10,9}+E_{13,11}\right), \\
x_{2, n}^{+}= & -q^{-1}\left(q^{-\alpha-3} z\right)^{n}\left(q^{n} E_{4,3}+q^{n} E_{7,5}+q^{-n} E_{12,10}+q^{-n} E_{14,13}\right), \\
x_{3, n}^{+}= & -q^{-1}\left(q^{-\alpha-3} z\right)^{n}\left(q^{2 n} E_{3,2}+E_{9,7}+E_{10,8}+q^{-2 n} E_{15,14}\right), \\
x_{4, n}^{+}= & \left(q^{-\alpha-3} z\right)^{n}\left(\sqrt{[\alpha]} q^{-\alpha+3 n} E_{2,1}+\sqrt{[\alpha+1]} q^{-\alpha-1+n}\left(E_{5,3}+E_{7,4}+E_{8,6}\right)\right. \\
& \left.+\sqrt{[\alpha+2]} q^{-\alpha-2-n}\left(E_{11,9}+E_{13,10}+E_{14,12}\right)+\sqrt{[\alpha+3]} q^{-\alpha-3-3 n} E_{16,15}\right), \\
x_{1, n}^{-}= & -q\left(q^{-\alpha-3} z\right)^{n}\left(E_{4,6}+E_{7,8}+E_{9,10}+E_{11,13}\right), \\
x_{2, n}^{-}= & -q\left(q^{-\alpha-3} z\right)^{n}\left(q^{n} E_{3,4}+q^{n} E_{5,7}+q^{-n} E_{10,12}+q^{-n} E_{13,14}\right), \\
x_{3, n}^{-}= & -q\left(q^{-\alpha-3} z\right)^{n}\left(q^{2 n} E_{2,3}+E_{7,9}+E_{8,10}+q^{-2 n} E_{14,15}\right), \\
x_{4, n}^{-}= & -\left(q^{-\alpha-3} z\right)^{n}\left(\sqrt{[\alpha]} q^{\alpha+3 n} E_{1,2}+\sqrt{[\alpha+1]} q^{\alpha+1+n}\left(E_{3,5}+E_{4,7}+E_{6,8}\right)\right. \\
& \left.+\sqrt{\alpha+n}\left(E_{9,11}+E_{10,13}+E_{12,14}\right)+\sqrt{[\alpha+3]} q^{\alpha+3-3 n} E_{15,16}\right) . \\
= & \\
= &
\end{aligned}
$$




\section{References}

[1] P.Bouwknegt, J.McCarthy and K.Pilch, Free field approach to 2-dimensional conformal field theories, Prog.Theor.Phys.102, 67-135, (1990).

[2] M.Jimbo and T.Miwa, Algebraic analysis of solvable lattice model, CBMS Regional Conference Series in Mathematics 85, AMS, 1984.

[3] T.Kojima, Free field realization of quantum affine superalgebra $U_{q}(\widehat{s l}(N \mid 1))$, J.Math.Phys.53, 013515 (1-15), (2012).

[4] T.Kojima, The $q$-Wakimoto realization of the superalgebras $U_{q, p}(\hat{s l}(N \mid 1))$ and $U_{q, p}(\widehat{s l}(N \mid 1))$, to appear in Proceedings of the 9th International Workshop "Lie Theory and its application in Physics 2011".

[5] M.Wakimoto, Fock Representations of the affine Lie algebra $A_{1}^{(1)}$, Commun.Math.Phys.104, no.4, 605-609 (1986).

[6] B.Feigin and E.Frenkel, Representations of affine Kac-Moody algebras and bosonization, Physics and Mathematics of Strings, World Scientific, Singapole, 271-316, (1990).

[7] A.Matsuo, A q-deformation of Wakimoto modules, primary fields and screening operators, Commun.Math.Phys.160, 33-48 (1994).

[8] J.Shiraishi, Free boson representation, Phys.Lett. A171, 243-248 (1992).

[9] H.Awata, S.Odake and J.Shiraishi, Free boson realization of $U_{q}\left(\widehat{s l}_{N}\right)$, Commun.Math.Phys. 162 no. 1, 61-83 (1994).

[10] H.Awata, S.Odake and J.Shiraishi, $q$-difference realization of $U_{q}(\operatorname{sl}(M \mid N))$ and its application to free boson realization of $U_{q}(\widehat{\mathrm{sl}}(2 \mid 1))$, Lett. Math. Phys.42 no. 3, 271-279 (1997).

[11] Y.-Z.Zhang and M.D.Gould, $U_{q}(\widehat{s l}(2 \mid 1))$ Vertex operators, screening currents and correlation functions at arbitrary level, J.Math.Phys.41, 5577-5291, (2000).

[12] I.B.Frenkel and V.G.Kac, basic representations of affine Lie algebras and dual resonance models, Invent.Math. 62, 23-66 (1980).

[13] G.Segal, Unitary representation of some infinite dimensional groups, Commun.Math.Phys.80, 301342 (1981).

[14] I.B.Frenkel and N.Jing, Vertex representations of quantum affine algebras, Proc.Natl.Acad.Sci. 85,9373-9377 (1988).

[15] D.Bernard, Vertex operator representations of quantum affine algebra $U_{q}\left(B_{r}^{(1)}\right)$, Lett.Math.Phys.17, 239-245 (1989). 
[16] N.Jing, Y.Koyama and K.Misra, Level one representations of quantum affine algebra $U_{q}\left(C_{n}^{(1)}\right), S e$ lecta Math.5, no. 2, 243-255 (1999).

[17] N.Jing, Twisted vertex representations of quantum affine algebras, Invent.Math.102, 663-690 (1990).

[18] N.Jing, Level one representations of $U_{q}\left(G_{2}^{(1)}\right)$, Proc.Amer.Math.Soc.127, no.1 21-27 (1999).

[19] K.Kimura, J.Shiraishi and J.Uchiyama, A level-one representation of the quantum affine superalgebra $U_{q}(\widehat{s l}(M+1 \mid N+1))$, Comm. Math. Phys. 188 no. 2, 367-378 (1997).

[20] Y.-Z.Zhang, Level-one representations and vertex operators of quantum affine superalgebra $U_{q}(\widehat{s l}(N \mid N))$, J.Math.Phys.40, no.11, 6110-6124 (1999).

[21] W.-L.Yang and Y.-Z.Zhang, Drinfeld basis and free boson representation of twisted quantum affine superalgebra $U_{q}\left(\operatorname{osp}(2 \mid 2)^{(2)}\right)$, Phys.Lett.A261, 252-258 (1999).

[22] P.Bouwknegt, A.Ceresole, J.G.McCarthy, and P.van Nieuwenhuizen, Extended Sugawara construction for the superalgebra $S U(M+1 \mid N+1)$. I. Free-field representation and bosonization of super Kac-Moody currents, Phys. Rev.D39, 2971-2986, (1989).

[23] Vl.S.Dotsenko and V.A.Fateev, Conformal algebra and multipoint correlation functions in 2d statistical models, Nuclear Phys.B240 no. 3, 312-348, (1984).

[24] H.Konno, BRST cohomology in quantum affine algebra $U_{q}\left(\widehat{s l}_{2}\right)$, Mod.Phys.Lett.A9, 1253-1265, (1994).

[25] A.Kato, Y.-H.Quano and J.Shiraishi, Free boson representation of $q$-vertex operators and their correlation functions, Comm. Math. Phys. 157 no. 1, 119-137, (1983).

[26] G.Felder, BRST approach to minimal model, Nucl.Phys.B317, 215-236, (1989).

[27] D.Bernard and G.Felder, Fock representations and BRST cohomology in $S L(2)$ current algebra, Commun.Math.Phys.127, 145-168, (1990).

[28] P.Bouwknegt, J.McCarthy and K.Pilch, Quantum group structure in the Fock space resolutions of $\widehat{s l}(n)$ representations, Commun. Math.Phys.131, 125-155, (1990).

[29] B.Feigin, M.Jimbo, T.Miwa, A.Odesskii and Ya.Pugai, Algebra of screening operators for the Deformed $W_{n}$ algebra, Commun.Math.Phys.191, 501-541, (1998).

[30] I.B.Frenkel and N.Yu.Reshetikhin, Quantum affine algebras and holonomic difference equations, Commun.Math.Phys.146,1-60, (1992).

[31] V.G.Kac, Lie superalgebras, Advances in Math.26 no. 1, 8-96 (1977).

[32] V.G.Kac, A sketch of Lie superalgebra theory, Comm. Math. Phys. 53 no. 1, 31-64 (1977). 
[33] V.G.Kac and M.Wakimoto, Integrable highest weight modules over affine superalgebras and number theory, Lie theory and geometry, Progr. Math.123, 415-456 (1994).

[34] H.Yamane, On defining relations of the affine Lie superalgebras and their quantized universal enveloping superalgebras, Publ.Res.Inst.Math.Sci. 35, 321-390, (1999).

[35] V.G. Drinfeld, A new realization of Yangians and quantized affine algebras, Sov.Math.Dokl. 36, 212-216, (1988).

[36] V.G.Kac, Representation of classical Lie superalgebras, Lect.Notes.in Math.676, 597-626, (1978).

[37] T.D.Palev and V.N.Tolstoy, Finite-dimensional irreducible representations of the quantum superalgebra $U_{q}(g l(n \mid 1))$, Commun.Math.Phys.141, 549-558, (1991). 